\title{
Meta-analysis of 208,370 East Asians identifies 113 genomic loci and yields new non-immune cell relevant biological insights for systemic lupus erythematosus
}

Xianyong Yin $1,2,3,4,5,6,66$, Kwangwoo Kim²,66, Hiroyuki Suetsugu, ${ }^{8,9,10,66}$, So-Young Bang ${ }^{11,12}$, Leilei Wen¹,2,3, Masaru Koido9,13, Eunji Ha7 , Lu Liu ${ }^{1,2,3}$, Yuma Sakamoto14, Sungsin $\mathrm{Jo}^{12}$, Rui-Xue Leng ${ }^{15}$, Nao Otomo ${ }^{8,9,16}$, Viktoryia Laurynenka ${ }^{17}$, Young-Chang Kwon $^{12}$, Yujun Sheng ${ }^{1,2,3}$, Nobuhiko Sugano ${ }^{18}$, Mi Yeong Hwang ${ }^{19}$, Weiran Li $^{1,2,3}$, Masaya Mukai ${ }^{20}$, Kyungheon Yoon ${ }^{19}$, Minglong Cai ${ }^{1,2,3}$, Kazuyoshi Ishigaki ${ }^{9,21,22,23}$, Won Tae Chung24, He Huang 1,2,3, Daisuke Takahashi25, Shin-Seok Lee ${ }^{26}$, Mengwei Wang $^{1,2,3}$, Kohei Karino ${ }^{27}$, Seung-Cheol Shim ${ }^{28}$, Xiaodong Zheng1,2,3, Tomoya Miyamura $^{29}$, Young Mo Kang ${ }^{30}$, Dongqing Ye ${ }^{15}$, Junichi Nakamura ${ }^{31}$, Chang-Hee Suh $^{32}$, Yuanjia Tang 33 , Goro Motomura ${ }^{10}$, Yong-Beom Park ${ }^{34}$, Huihua Ding 33 , Takeshi Kuroda ${ }^{35}$, Jung-Yoon Choe ${ }^{36}$, Chengxu Li ${ }^{5}$, Hiroaki Niiro ${ }^{37}$, Youngho Park ${ }^{12}$, Changbing Shen ${ }^{38,39}$, Takeshi Miyamoto ${ }^{40}$, Ga-Young Ahn ${ }^{11}$, Wenmin Fei ${ }^{5}$, Tsutomu Takeuchi ${ }^{41}$, Jung-Min Shin ${ }^{11}$, Keke Li ${ }^{5}$, Yasushi Kawaguchi ${ }^{42}$, Yeon-Kyung Lee ${ }^{11}$, Yongfei Wang ${ }^{43}$, Koichi Amano ${ }^{44}$, Wanling Yang ${ }^{43}$, Yoshifumi Tada ${ }^{45}$, Ken Yamaji ${ }^{46}$, Masato Shimizu ${ }^{47}$, Takashi Atsumi ${ }^{48}$, Akari Suzuki ${ }^{49}$, Takayuki Sumida ${ }^{50}$, Yukinori Okada $^{51,52}$, Koichi Matsuda ${ }^{53,54}$, Keitaro Matsuo ${ }^{55,56}$, Yuta Kochi ${ }^{57}$, Japanese Research Committee on Idiopathic Osteonecrosis of the Femoral Head ${ }^{*}$ Leah C. Kottyan ${ }^{17,58}$, Matthew T. Weirauch ${ }^{17,58}$, Sreeja Parameswaran ${ }^{17}$, Shruti Eswar ${ }^{17}$, Hanan Salim ${ }^{17}$, Xiaoting Chen ${ }^{17}$, Kazuhiko Yamamoto ${ }^{49}$, John B. Harley ${ }^{17,58,59}$, Koichiro Ohmura ${ }^{60}$, Tae-Hwan Kim ${ }^{11,12}$, Sen Yang $1,2,3$, Takuaki Yamamoto ${ }^{61}$, Bong-Jo Kim ${ }^{19}$, Nan 
Shen ${ }^{17,33,62}$, Shiro Ikegawa ${ }^{8}$, Hye-Soon Lee ${ }^{11,12}$, Xuejun Zhang ${ }^{1,2,3,63}$, Chikashi Terao $9,64,65,67$, Yong Cui 5,67 , Sang-Cheol Bae11,12,67

${ }^{1}$ Department of Dermatology, First Affiliated Hospital, Anhui Medical University, Hefei, Anhui, China

${ }^{2}$ Institute of Dermatology, Anhui Medical University, Hefei, Anhui, China

${ }^{3}$ Key Lab of Dermatology, Ministry of Education (Anhui Medical University), Hefei, Anhui, China

${ }^{4}$ Inflammation and Immune Mediated Diseases Laboratory of Anhui Province, Hefei, Anhui, China

${ }^{5}$ Department of Dermatology, China-Japan Friendship Hospital, Beijing, China ${ }^{6}$ Department of Biostatistics, Center for Statistical Genetics, University of Michigan, Ann Arbor, MI, USA

${ }^{7}$ Department of Biology and Department of Life and Nanopharmaceutical Sciences, Kyung Hee University, Seoul, Korea

${ }^{8}$ Laboratory for Bone and Joint Diseases, RIKEN Center for Medical Sciences, Kanagawa, Japan

${ }^{9}$ Laboratory for Statistical and Translational Genetics Analysis, RIKEN Center for Integrative Medical Sciences, Kanagawa, Japan

${ }^{10}$ Department of Orthopaedic Surgery, Graduate School of Medical Sciences, Kyushu University, Fukuoka, Japan

${ }^{11}$ Department of Rheumatology, Hanyang University Hospital for Rheumatic Diseases, Seoul, Korea 
${ }^{12}$ Hanyang University Institute for Rheumatology Research, Seoul, Korea

${ }^{13}$ Division of Molecular Pathology, Department of Cancer Biology, Institute of

Medical Science, The University of Tokyo, Tokyo, Japan

${ }^{14}$ Koga Hospital 21, Japan

${ }^{15}$ Department of Epidemiology and Biostatistics, School of Public Health, Anhui

Medical University, Hefei, Anhui, China

16Department of Orthopedic Surgery, Keio University School of Medicine, Tokyo,

Japan

${ }^{17}$ Center for Autoimmune Genomics and Etiology (CAGE), Cincinnati Children's Hospital Medical Center, Cincinnati, Ohio, USA

${ }^{18}$ Department of Orthopaedic Medical Engineering, Osaka University Graduate School of Medicine, Osaka, Japan

${ }^{19}$ Division of Genome Research, Center for Genome Science, National Institute of Health, Osong Health Technology Administration Complex, Cheongju, Korea

${ }^{20}$ Department of Rheumatology \& Clinical Immunology, Sapporo City General Hospital, Hokkaido, Japan

${ }^{21}$ Divisions of Genetics and Rheumatology, Department of Medicine, Brigham and Women's Hospital, Harvard Medical School, Boston, MA, USA

${ }^{22}$ Center for Data Sciences, Harvard Medical School, Boston, MA, USA

23Program in Medical and Population Genetics, Broad Institute of MIT and Harvard, Cambridge, MA, USA

${ }^{24}$ Department of Internal Medicine, Dong-A University Hospital, Busan, Korea 
${ }^{25}$ Department of Orthopaedic Surgery, Faculty of Medicine and Graduate School of Medicine, Hokkaido University, Hokkaido, Japan

${ }^{26}$ Division of Rheumatology, Department of Internal Medicine, Chonnam National University Medical School and Hospital, Gwangju, Korea

27Department of Rheumatology, Endocrinology and Nephrology, Faculty of Medicine and Graduate School of Medicine, Hokkaido University, Hokkaido, Japan

${ }^{28}$ Division of Rheumatology, Department of Internal Medicine, Chungnam National University Hospital, Daejeon, Korea

${ }^{29}$ Department of Internal Medicine and Rheumatology, National Hospital

Organization, Kyushu Medical Center, Fukuoka, Japan

${ }^{30}$ Division of Rheumatology, Department of Internal medicine, Kyungpook National University Hospital, Daegu, Korea

${ }^{31}$ Department of Orthopaedic Surgery, Graduate School of Medicine, Chiba University, Chiba, Japan

32Department of Rheumatology, Ajou University School of Medicine, Suwon, Korea ${ }^{33}$ Shanghai Institute of Rheumatology, Renji Hospital, Shanghai Jiao Tong University, School of Medicine (SJTUSM), Shanghai, China

${ }^{34}$ Department of Internal Medicine, Yonsei University College of Medicine, Seoul, Korea

${ }^{35}$ Niigata University Health Administration Center, Niigata, Japan

${ }^{36}$ Department of Rheumatology, Catholic University of Daegu School of Medicine, Daegu, Korea 
${ }^{37}$ Department of Medical Education, Kyushu University Graduate School of Medical Sciences, Fukuoka City, Japan

${ }^{38}$ Department of Dermatology, Peking University Shenzhen Hospital, Shenzhen, Guangdong, China

${ }^{39}$ Shenzhen Key Laboratory for Translational Medicine of Dermatology, Shenzhen Peking University - The Hong Kong University of Science and Technology Medical Center, Shenzhen, Guangdong, China

${ }^{40}$ Department of Orthopaedic Surgery, Faculty of Life Sciences, Kumamoto

University, Kumamoto, Japan

${ }^{41}$ Division of Rheumatology, Department of Internal Medicine, Keio University

School of Medicine, Tokyo, Japan

${ }^{42}$ Institute of Rheumatology, Tokyo Women's Medical University, Tokyo, Japan

${ }^{43}$ Department of Paediatrics and Adolescent Medicine, The University of Hong Kong,

Pok Fu Lam, Hong Kong, China

${ }^{44}$ Department of Rheumatology \& Clinical Immunology, Saitama Medical Center,

Saitama Medical University, Saitama, Japan

${ }^{45}$ Department of Rheumatology, Faculty of Medicine, Saga University, Saga, Japan

${ }^{46}$ Department of Internal Medicine and Rheumatology, Juntendo University School

of Medicine, Tokyo, Japan

${ }^{47}$ Hokkaido Medical Center for Rheumatic Diseases, Japan

${ }^{48}$ Department of Orthopaedic Surgery, Showa University School of Medicine, Tokyo, Japan 
${ }^{49}$ Laboratory for Autoimmune Diseases, RIKEN Center for Integrative Medical

Sciences, Kanagawa, Japan

${ }^{50}$ Department of Internal Medicine, Faculty of Medicine, University of Tsukuba, Ibaraki, Japan

${ }^{51}$ Department of Statistical Genetics, Osaka University Graduate School of Medicine, Osaka, Japan

${ }^{52}$ Laboratory of Statistical Immunology, Immunology Frontier Research Center (WPi-iFReC), Osaka University, Osaka, Japan

${ }^{53}$ Laboratory of Genome Technology, Human Genome Center, Institute of Medical Science, The University of Tokyo, Tokyo, Japan

${ }^{54}$ Laboratory of Clinical Genome Sequencing, Department of Computational Biology and Medical Sciences, Graduate School of Frontier Sciences, The University of Tokyo, Tokyo, Japan

55Division of Cancer Epidemiology and Prevention, Aichi Cancer Center Research Institute, Nagoya, Japan

${ }^{56}$ Department of Epidemiology, Nagoya University Graduate School of Medicine, Nagoya, Japan

${ }^{57}$ Department of Genomic Function and Diversity, Medical Research Institute, Tokyo Medical and Dental University, Tokyo, Japan

${ }^{58}$ Department of Pediatrics, University of Cincinnati, Cincinnati, Ohio, USA

${ }^{59}$ US Department of Veterans Affairs Medical Center, Cincinnati, Ohio, USA

${ }^{60}$ Department of Rheumatology and Clinical immunology, Kyoto University Graduate school of Medicine, Kyoto, Japan 
${ }^{61}$ Department of Orthopaedic Surgery, Faculty of Medicine, Fukuoka University, Fukuoka, Japan

${ }^{62}$ State Key Laboratory of Oncogenes and Related Genes, Shanghai Cancer Institute, Renji Hospital, Shanghai Jiao Tong University School of Medicine (SJTUSM), Shanghai, China

${ }^{63}$ Department of Dermatology, Institute of Dermatology, Huashan Hospital, Fudan University, Shanghai, China

${ }^{64}$ Clinical Research Center, Shizuoka General Hospital, Shizuoka, Japan

65The Department of Applied Genetics, The School of Pharmaceutical Sciences, University of Shizuoka, Shizuoka, Japan

${ }^{*}$ A full list of members and affiliations appears in supplementary material

${ }^{66}$ These authors contributed equally: Xianyong Yin, Kwangwoo Kim, and Hiroyuki Suetsugu

${ }^{67}$ These authors jointly supervised this research: Chikashi Terao, Yong Cui, and Sang-Cheol Bae. 


\section{Abstract (150 words):}

Systemic lupus erythematosus (SLE), an autoimmune disorder, has been associated with nearly 100 susceptibility loci1-8. Nevertheless, these loci only partially explain SLE heritability and provide limited biological insight. We report the largest study of SLE in East Asians (13,377 cases and 194,993 controls), identifying 233 association signals within 113 (46 novel) genetic loci. We detect six new lead missense variants and prioritize ten most likely putative causal variants, one of which we demonstrate exhibits allele-specific regulatory effect on ACAP1 in vitro. We suggest 677 effector genes with potential for drug repurposing, and provide evidence that two distinct association signals at a single locus act on different genes (NCF2 and SMG7). We demonstrate that SLE-risk variants overlap with cell-specific active regulatory elements, notably EBNA2-mediated super-enhancers in Epstein-Barr Virustransformed B cells, and implicate the role for non-immune cells in SLE biology. These findings shed light on genetic and biological understandings of SLE. 


\section{Main text}

Systemic lupus erythematosus (SLE) is an autoimmune disorder characterized by the production of autoantibodies that damage multiple organs ${ }^{9}$. Considerable genetic predisposition contributes to SLE etiology ${ }^{10}$. To date, nearly 100 susceptibility loci have been identified for SLE, mainly through genome-wide association studies (GWASs) ${ }^{1-4,7,8}$. However, these loci collectively only explain $\sim 30 \%$ of SLE heritability ${ }^{5}$ and their biology, in terms of causal variants, effector genes and cell types, and pathological pathways that mediate genetic effects, has not yet been fully characterized ${ }^{11}$.

Genome-wide association meta-analyses (GWMA) have been performed to uncover new genetic associations for SLE in Asians ${ }^{6}$, Europeans ${ }^{12}$, and transancestral populations ${ }^{5}$. However, the study sample sizes were relatively modest, which limits their ability for genetic discovery. GWASs have successfully linked genetic variants with human common diseases and traits ${ }^{13}$. Nonetheless, only $\sim 8 \%$ of GWAS participants are East Asians ${ }^{14}$. East Asians have a unique population genetic history and may have unique genetic disease risk mechanisms and exhibit specific disease manifestations. For example, SLE has a remarkably higher prevalence and younger age of onset in Asians ${ }^{15,16}$. Genetic heterogeneity may explain, at least partly, the phenotypic diversity of SLE between East Asians and Europeans ${ }^{5}$. Hence, large-scale East Asian investigations may identify unique genetic associations even for the same diseases and traits that have already been well studied in Europeans ${ }^{17}$. 
Here, we report the largest-ever GWMA for SLE in 208,370 East Asians. Our study identified many new genetic associations, some of which have not been detected in Europeans, and improved our biological understanding of SLE pathophysiology.

We newly generated three GWAS datasets from 10,029 SLE cases and 180,167 controls, which we meta-analyzed together with five published studies $(3,348 \text { cases and } 14,826 \text { controls })^{1,2,4,8,18}$ after whole-genome genotype imputation using 1000 Genomes Project phase $3^{19}$ and population-specific reference panels ${ }^{20}$, bringing the total sample size to 208,370 (Supplementary Table 1). To the best of our knowledge, this is the largest genetic association study of SLE to date. The effective sample size $\left(\mathrm{N}_{\mathrm{eff}}=50,072\right)$ is three- and four-fold larger than that of the largest published trans-ancestry ${ }^{5}$ and East Asian ${ }^{6}$ meta-analyses, respectively.

We tested associations for 11,270,530 genetic variants in a fixed-effects meta-analysis. A quantile-quantile plot showed that test statistics were wellcalibrated, with a genomic-control inflation factor $\lambda_{\mathrm{GC}}=1.06$ (indicating that ancestry effects had been well controlled; Supplementary Figure 1). Linkage disequilibrium (LD) score regression ${ }^{21}$ showed that polygenic effects (89.4\%), rather than biases, primarily caused the inflation residual (estimated mean $\chi^{2}=1.32$ and LD-score intercept=1.03).

We detected 26,379 genetic variants associated with SLE at $\mathrm{P}<5 \times 10^{-8}$ within 113 loci (Supplementary Table 2 and Fig. 1a), of which 46 were novel (Tab. 1). The pairwise LD between lead variants is low ( $\left.L D r^{2}<0.002\right)$. For seven novel loci, minor allele frequencies (MAFs) of the lead single nucleotide polymorphisms (SNPs) 
medRxiv preprint doi: https://doi.org/10.1101/2020.08.22.20178939; this version posted August 25, 2020. The copyright holder for this preprint (which was not certified by peer review) is the author/funder, who has granted medRxiv a license to display the preprint in perpetuity.

All rights reserved. No reuse allowed without permission.

were ten-fold higher in East Asians than in Europeans (Fig. 1b). Two of them and their neighbors in strong LD (LD ${ }^{2} \geq 0.2$ in either East Asians or Europeans) would be undetectable in Europeans at the same effective sample size and risk magnitude as we find in East Asians (statistical power<10\%; Supplementary Table 3).

To dissect the source of association signals at each locus, we conducted an approximate conditional analysis using $\mathrm{GCTA}^{22}$ with meta-analysis summary statistics and LD estimates from 7,021 unrelated Chinese controls (Online Methods). We acknowledge the limitations of using LD estimation from a single population for a meta-analysis of diverse East Asians. We identified a total of 233 independent association signals with conditional $\mathrm{P}<5 \times 10^{-8}, 169$ of which arose from non-Human Leukocyte Antigen (HLA) regions (Supplementary Table 4). We observed two to four signals at each of 28 non- $H L A$ loci (including 7 novel loci). For example, we discovered two distinct association signals within the known STAT4 locus, including the previously reported SNP rs $11889341^{12}$ and the new insertdeletion variant (indel) rs71403211 (Extended Data Fig. 1a). For the 46 novel loci, we discovered 55 distinct signals (Supplementary Table 4 and Supplementary Figure 2). Most of the signal index variants ( $n=190,82 \%)$ are common $(M A F \geq 5 \%)$ with modest effects (Supplementary Table 4).

We identified 11 exonic signal index variants at ten non- $H L A$ loci (Supplementary Table 4), highlighting the roles of $A H N A K 2, C S K, I K B K B, I R A K 1$, NCF2, OAS1, TYK2, and WDFY4 within eight known loci, and CDH23 and LRRK1 within two novel loci (Extended Data Fig. 1-2). We detected two distinct signals within WDFY4, including the known (rs7097397) ${ }^{18}$ and a new (rs7072606) 
missense variant (LD r ${ }^{2}=0.02$ in East Asians), which suggest a potential allelic series effect at this locus (Extended Data Fig. 1b). We replicated the association of the missense variants at AHNAK2 (rs2819426) ${ }^{23}$, IRAK1 (rs1059702) ${ }^{24}$, and NCF2 (rs13306575) ${ }^{25,26}$, and provided for the first time genome-wide association evidence at a missense variant within $O A S 1$ (rs1131476, LD $\mathrm{r}^{2}=0.78$ with the known missense variant rs1051042 that attains suggestive significance in East Asians) ${ }^{23}$. We detected three new exonic variants (including two missense variants) within the CSK (rs11553760), IKBKB (rs2272736), and TYK2 (rs55882956) genes (Extended Data Fig. 2). They were not correlated with previously reported exonic variants within the same genes (LD r ${ }^{2}<0.02$ in East Asians or Europeans; Supplementary Table 5), suggesting possible allelic heterogeneity of these genes. In the two novel loci with lead missense variants (Extended Data Fig. 2), CHD23 plays a role in cell migration ${ }^{27}$ while $L R R K 1$ encodes a multiple-domain leucine-rich repeat kinase. A previous study observed that $L R R K 1$-deficient mice exhibited a profound defect in B-cell proliferation and survival and impaired B-cell receptor-mediated NF- $\mathrm{B}$ activation $^{28}$.

To prioritize putative causal variants, we conducted a Bayesian statistical fine-mapping analysis for 111 loci using FINEMAP ${ }^{29}$ after excluding complex associations involving $H L A$ and $7 \mathrm{q} 11.23$. We found exactly the same number of association signals in 57 loci between FINEMAP causal configuration with the highest posterior probability (PP) and the GCTA approximate conditional test. To be conservative, we only summarized the statistical fine-mapping results for these 57 regions, which contained 65 association signals (Supplementary Table 6). 
For each signal, we built a credible set of putative causal variants with a $95 \%$ probability of including the true causal variants. The size of 28 credible sets was small (size $\leq 10$; Fig. 2a). Among the 110 putative causal variants with $P P \geq 0.1$ (Fig. 2b), we found four coding variants (3.6\%), which implies that most of these associations are probably induced by non-coding causal variants. The prioritized variants are available to be tested as potential targets in perturbation experiments. For example, the allele-specific regulatory activity of the intronic variant (rs10036748) with the highest PP (0.387) in the TNIP1 locus was recently characterized in $\mathrm{SLE}^{30}$.

We pinpointed a single most likely causal variant with high confidence (PP $\geq 0.8$ ) for four known (ATXN2, BACH2, DRAM1/WASHC3, and NCF2) and six novel (17p13.1, ELF3, GTF2H1, LRRK1, LOC102724596/PHB, and STIM1) loci (Supplementary Table 6). For example, we prioritized rs61759532 as a putative causal variant at the novel 17p13.1 locus ( $\mathrm{PP}=0.999$; Fig. 3a). This variant is located in an intron of $A C A P 1$, which encodes a key regulator of integrin traffic for cell adhesion and migration ${ }^{31}$. We observed that rs61759532 overlaps with an accessible open chromatin region in blood B and T cells (Fig. 3b). Transcriptional reporter assays showed significant allelic differences in the enhancer activity of rs61759532 in THP1 monocyte cell lines (two-sided t-test P=8.1×10-3; Fig. 3c), consistent with the regulatory effect of the risk allele, $T$, in reducing $A C A P 1$ expression levels in whole blood ${ }^{32}\left(\mathrm{P}=1.7 \times 10^{-47}\right.$; Fig. 3d). Electrophoretic mobility shift assays (EMSA) revealed that allele-specific biotin-labeled probes containing the 
$T$ (risk allele) form fewer nuclear protein-probe complexes than probes with $C$ (nonrisk allele) in THP1 and Epstein-Barr virus (EBV)-transformed B cell lines (Fig. 3e).

We deployed four gene-level methods to comprehensively catalog potential effector genes. Briefly, we performed a transcriptome-wide association study (TWAS) ${ }^{33}$ using expression quantitative trait loci (eQTL) from six human immune cell types in up to 105 East Asians $^{34}$, a gene-based association analysis using Multimarker Analysis of GenoMic Annotation (MAGMA) ${ }^{35}$, and two additional datamining approaches ${ }^{36,37}$ that integrated genetic associations with gene function and chromosomal position, eQTL, and chromatin interactions (Online Methods). We nominated 677 possible effector genes (Supplementary Table 7-11 and Extended Data Fig. 3). Of the 677 genes, 285 (42\%) were supported by at least two approaches and 222 (35.4\%) were predicted to have differential expression levels in SLE in blood immune cells from East Asian individuals, including 26 genes from regions $(<500 \mathrm{~kb})$ where genome-wide significant associations have not previously been reported. For example, we found significant evidence for the $F A S$ gene only by TWAS. This gene encodes a member of the TNF receptor superfamily and plays a central role in lymphocyte apoptosis. Individuals with defects in the Fas/FasL system develop lupus-like symptoms ${ }^{38}$.

The gene-level analysis provided an opportunity to interrogate the biology of SLE loci. For example, we detected two independent association signals within the known SLE locus including SMG7 and NCF2 (Fig. 4a). The lead SNP rs13306575 is a missense variant, which substitutes arginine to tryptophan at NCF2 and then disrupts the NADPH oxidase complex, nominating NCF2 as an effector gene (Fig. 
4b). The secondary variant rs66977652 ( $~ 8 \mathrm{~kb}$ away from rs13306575, pairwise LD $r^{2}=0.02$ ) resides in an intron of $N C F 2$. Our gene-level analysis showed that rs66977652 confers a significant eQTL effect on $S M G 7\left(\mathrm{P}=3.1 \times 10^{-15}\right.$ in whole blood; Fig. 4c and Supplementary Figure 3), suggesting the secondary signal might influence the risk of SLE by modulating SMG7 expression, which is supported by functional studies ${ }^{39}$. These findings suggest two potential effector genes, NCF2 and SMG7, in a single locus ${ }^{25,26}$. As another example, a single-variant association test failed to support one effector gene for a novel locus that contains seven proteincoding genes (lead variant rs 11288784; Supplementary Figure 2). But we found that only HEATR3 achieved significance in both MAGMA gene-based association analysis and TWAS (Supplementary Table 10-11). HEATR3 may modulate SLE risk through NOD2-mediated NF- $\kappa B$ signaling ${ }^{40}$. Altogether, the 677 genes implicate roles for cytokine production and signaling, immune responses to stimuli, and the phosphorus metabolic pathway in SLE pathogenesis (Supplementary Table 12), and might inform repurposing drugs approved for musculoskeletal system disorders (Supplementary Table 13).

To assess the proportion of phenotypic variance explained by common variants, we applied LD score regression ${ }^{21}$ to the meta-analysis results. Assuming a population prevalence of $0.03 \%$ for SLE $^{9}$, we estimated the liability-scale SNP-based heritability from all non- $H L A$ variants as $\mathrm{h}^{2} \mathrm{SNP}$ ? =? 7.24\% (standard error $(\mathrm{SE})=0.78 \%$ ). The 66 known and 46 novel non-HLA loci explained $62.6 \%$ (SE=4.9\%) and $22.1 \%$ (SE=2.6\%) of this overall SNP-based heritability, respectively. 
medRxiv preprint doi: https://doi.org/10.1101/2020.08.22.20178939; this version posted August 25, 2020. The copyright holder for this preprint (which was not certified by peer review) is the author/funder, who has granted medRxiv a license to display the preprint in perpetuity.

All rights reserved. No reuse allowed without permission.

To evaluate global enrichment for SLE-associated variants in epigenomic features, we annotated SLE-associated variants with 15 Roadmap chromatin states in various immune cells using Genomic Regulatory Elements and Gwas Overlap algoRithm (GREGOR) ${ }^{41}$. SLE-associated variants were most significantly enriched in transcription-activating chromatin states at transcription start sites (TSSs) and enhancers (Fig. 5a and Supplementary Table 14).

To identify tissues and cell types in which SLE-associated genes impact, we tested for expression of SLE-associated genes across human tissues using Datadriven Expression-Prioritized Integration for Complex Traits (DEPICT) ${ }^{36}$. We found significant enrichment for SLE-associated genes not only in the hemic and immune system $\left(\mathrm{P}<1.24 \times 10^{-3}\right)$ but also in musculoskeletal, respiratory, stomatognathic, and digestive tissues (Fig. 5b, Supplementary Figure 4, and Supplementary Table 15). This might be related with various complication symptoms in multiple organs. In order to gain further mechanistic insight into non-coding risk variant, we used our RELI method ${ }^{42}$ to identify specific transcription factors (TFs) that significantly occupy SLE risk loci. Eighty-eight TFs concentrate their DNA binding in the 113 SLE loci (relative risk (RR)=2.1-19.0, $P<10^{-6}$; Supplementary Table 16), 40 loci of which (35.4\%; including 17 novel loci) were occupied by the EBV Nuclear Antigen 2 (EBNA2) protein $\left(\mathrm{RR}=4.9, \mathrm{P}=3.95 \times 10^{-30}\right.$; Fig. 5c and Extended Data Fig. 4), which is encoded during the EBV Latency III program, and contributes to B cell transformation. This study achieved a similar finding from independently ascertained SLE cases of East Asian origin, compared with a previous study of Europeans (Supplementary Table 17) ${ }^{42}$. In addition, the 46 novel loci were 
independently associated with EBNA2 (RR=5.0, $\mathrm{P}<1.7 \times 10^{-12}$; Supplementary Table 18). Significantly-associated TF ChIP-seq datasets are enriched for EBV-infected B cell lines, relative to EBV-negative cells (odds ratio $(O R)=11.1, P=4.33 \times 10^{-55}$;

Supplementary Table 19). The TFs present at super-enhancers formed upon EBV infection $^{43}$ were remarkably enriched in EBV-positive B cells relative to not EBVinfected cell (OR=22, $\mathrm{P}=0.001$; Supplementary Table 20-22). These results support a role for a gene-environment interaction between SLE loci and EBV infection. We further characterized significant heritability enrichments within 148 cell-state-specific TF profiles for 72 TFs and 123 types of cell or tissue types ${ }^{44}$ (FDR <5\%; Supplementary Table 23), which highlight the biological involvement of B cells in SLE pathophysiology.

To identify possible gene regulatory mechanisms impacted by non-coding SLE variants, we performed allelic read imbalance analysis on 985 publicly available ChIP-seq experiments performed in lymphoblast EBV-immortalized B cell lines (see Online Methods). Of the 113 SLE loci, 46 were heterozygous in genotyped cell lines, and 28 exhibited allelic imbalance in at least one ChIP-seq dataset (Supplementary Table 24). The active chromatin histone marks, H3K27ac and H3K4me1, showed strong imbalance in 13 and 8 loci, respectively. For several variants, we observed strong allelic imbalance for particular TFs, suggesting allele-specific function and an underlying genetic mechanism. For example, we observed allelic imbalance at rs2205960 near TNFSF4, with EP300, MLLT1, ARID3A, NFATC3, BACH1, MTA2, IKZF1, and BATF all preferring the "G" reference allele, and RUNX3, SMARCA5, 
TCF12, JUNB, NBN, SKIL, and POU2F2, all preferring the "T" non-reference allele (Extended Data Fig. 5).

To explore cell type-specific regulatory mechanism for non-coding variants, we applied a machine learning approach to predict variants' mutation effects in 347 types of primary cells and tissues ${ }^{45}$. We identified 71 variant-gene-cell sets showing robust mutation effects on gene transcription in immune cells (Supplementary Table 25). The alternative alleles of rs77571059 and rs200489061 have mutation effects on up- and down-regulating $I R F 5$ and $B L K$ expressions, respectively (Extended Data Fig. 6), both of which are consistent with previous in vitro experiments ${ }^{46,47}$.

To explore shared genetics between SLE and various traits, we calculated genetic correlations of SLE with 39 complex diseases and 59 quantitative traits in Biobank Japan participants using bivariate LD score regression ${ }^{48}$ (Supplementary Table 26). As expected, we detected significant positive genetic correlations between SLE and two other autoimmune diseases: rheumatoid arthritis $\left(\mathrm{r}_{\mathrm{g}}=0.437\right)$ and Graves' disease $\left(\mathrm{r}_{\mathrm{g}}=0.318\right)$. In addition, we found unreported genetic correlations $(\mathrm{FDR}<0.05)$ with albumin/globulin ratio $\left(\mathrm{r}_{\mathrm{g}}=-0.242\right)$ and non-albumin protein $\left(r_{g}=0.238\right)$. These findings may reflect the renal complications in SLE patients who have been reported to have significantly lower albumin/globulin ratio and higher serum globulin than healthy controls in epidemiological studies ${ }^{49}$.

We performed the largest GWMA for SLE in East Asians, reiterating the benefits of investigating genetic predispositions to SLE in less-studied populations. We illustrate the advantages of using genetic data to improve knowledge of disease 
medRxiv preprint doi: https://doi.org/10.1101/2020.08.22.20178939; this version posted August 25, 2020. The copyright holder for this preprint (which was not certified by peer review) is the author/funder, who has granted medRxiv a license to display the preprint in perpetuity.

All rights reserved. No reuse allowed without permission.

genes, effector tissues, regulatory mechanisms, and biological pathways involved in SLE etiology. These findings elucidate many aspects of SLE genetics and biology and have implications for precision health in SLE. 
medRxiv preprint doi: https://doi.org/10.1101/2020.08.22.20178939; this version posted August 25, 2020. The copyright holder for this preprint (which was not certified by peer review) is the author/funder, who has granted medRxiv a license to display the preprint in perpetuity.

\section{Online Methods}

Genome-wide association analyses in eight SLE case-control data sets. We newly recruited 10,029 SLE cases and 180,167 controls in three independent sets and genotyped them on the Illumina Infinium 0mniExpress Exome-8 Array, Illumina Infinium Global Screening Array, or Korean Biobank Arrays ${ }^{50}$ (Supplementary Table 1). Cases were diagnosed by medical specialists using American College of Rheumatology classification criteria for SLE 51 . Controls had neither SLE nor family history of SLE. Written informed consent was obtained from all participants. Protocols were approved by institutional review boards in participating institutions.

To improve statistical power for discovering genetic effects, we revisited raw genome-wide genotypes from five published studies ${ }^{1,2,4,5,8}$ (Supplementary Table 1). Quality controls were then conducted for each of the eight data sets. Briefly, we excluded individuals of: 1 . call rate $<95 \%$; 2 . mismatch between ascertained and genotype-inferred sex; 3. outliers for heterozygosity rate; 4. population outliers from the East Asian cluster in principal component analysis (PCA) of genotypes against 1000 Genomes Project (1KGP) populations ${ }^{19}$. For quality controls of genetic variants, we excluded variants with any of the following criteria: 1 . call rate $<99 \%$ for Japanese data sets or $<95 \%$ for the remainder; 2. P value for Hardy-Weinberg equilibrium $\left(\mathrm{P}_{\mathrm{HWE}}\right)<1.0 \times 10^{-6}$ in the controls; 3 . minor allele counts $(\mathrm{MAC})<10$ for Japanese data sets and $M A F \leq 1 \%$ for the others. We then conducted genotype imputation for each data set separately. Haplotypes were estimated using SHAPEIT ${ }^{52}$ or Eagle $2^{53}$. Genotype imputation was accomplished using reference panels from the $1 \mathrm{KGP}$ phase $3 \mathrm{v} 5^{19}$ and IMPUTE2 $/ 4^{54,55}$, or MINIMAC456. For 
medRxiv preprint doi: https://doi.org/10.1101/2020.08.22.20178939; this version posted August 25, 2020. The copyright holder for this preprint (which was not certified by peer review) is the author/funder, who has granted medRxiv a license to display the preprint in perpetuity.

All rights reserved. No reuse allowed without permission.

genotype imputation in individuals from Korea and Japan, we additionally used population-specific reference panels from 397 Korean Reference Genome Project ${ }^{20}$ and 7,472 whole-genome sequencing datasets, respectively.

We tested association between SLE risk and genotype dosages in each data set using a logistic regression or linear mixed model in PLINK57, SNPTEST ${ }^{58}$, or EPACTS (https://genome.sph.umich.edu/wiki/EPACTS) (Supplementary Table 1). Within each data set, we filtered out association results based on imputation quality (IMPUTE info or MINIMAC $\mathrm{r}^{2} \leq 0.3$ ), $\mathrm{MAF} \leq 0.5 \%$, or $\mathrm{P}_{\mathrm{HWE}}<1.0 \times 10^{-6}$. For each cohort, the association analysis for the $\mathrm{X}$ chromosome was conducted separately by sex and then meta-analyzed across both men and women. For data sets analyzed using a linear mixed model (Supplementary Table 1), allelic effects and standard errors were converted to a log-odds scale to correct for case-control imbalance ${ }^{59}$.

Fixed-effects meta-analysis. We aggregated the association summary statistics from the eight data sets using a fixed-effects inverse-variance meta-analysis in METAL $^{60}$. We applied a genomic control correction to each association summary statistic. Heterogeneity in allelic effect sizes among data sets was assessed using Cochran's Q statistic. We excluded genetic variants available in only a single data set. We defined SLE susceptibility loci by merging $\pm 250 \mathrm{~kb}$ windows around genomewide associated variants to ensure that lead SNPs were at least $500 \mathrm{~kb}$ apart. We defined lead variants as the most significant SLE-associated variant within each locus. A locus was considered novel if the lead SNP was at least $500 \mathrm{~kb}$ away from any previously reported SLE-associated variants. 
Approximate conditional association analysis. To dissect distinct association signals at each SLE locus, we performed an approximate conditional analysis using GCTA $\mathrm{COJO}^{22}$ with genome-wide meta-analysis summary statistics based on LD estimated from 7,021 unrelated Chinese controls. The Chinese reference individuals for LD calculation were retrieved from the Chinese study using the Illumina Infinium Global Screening Array data (Supplementary Table 1), excluding firstand second-degree relatives. We excluded genetic variants that have substantial MAF differences $(>0.05)$ between meta-analysis summary statistics and the reference individuals. We only presented signals below a conditional threshold of $\mathrm{P}<5 \times 10^{-8}$

Bayesian statistical fine-mapping analysis. To prioritize causal variants in SLE susceptibility loci, a statistical fine-mapping analysis was performed using FINEMAP v1.4 software ${ }^{29}$, with meta-analysis z-scores and LD matrices estimated from the 7,021 Chinese reference individuals. We used default priors and parameters in FINEMAP, assuming at most five causal signals in the $\pm 250 \mathrm{~kb}$ region around a lead variant at each SLE locus, excluding the HLA region (chromosome 6: 25-34 Megabases (Mb) in build hg19) and 7q11.23. FINEMAP was used to estimate the PPs and Bayes factor values of potential causal configurations and variant-level PPs for causality calculated for individual variants. We then built the $95 \%$ posterior credible sets of causal variants. The causal configurations were prioritized based on configuration-level PPs. 


\section{Assay for transposase-accessible chromatin using sequencing (ATAC-seq) in}

blood CD4+ T and CD19+ B cells. We collected fresh whole blood samples from five healthy volunteers of Chinese population. $\mathrm{CD}^{+} \mathrm{T}$ and $\mathrm{CD} 19^{+} \mathrm{B}$ cells were sorted using fluorescence activated cell sorting and used to create ATAC-seq libraries as previously described ${ }^{61}$. Libraries were sequenced on the BGISEQ 500 platform, with a $50 \mathrm{bp}$ paired-end read (unpublished data) and ATAC-seq peaks were called using MACS2 ${ }^{62}$, to detect open accessible elements at the ACAP1 locus in $\mathrm{CD}^{+} \mathrm{T}$ and CD19+ B cells. Each participant provided written consent. The study protocol was approved by the institutional review board at the Institution of Dermatology, Anhui Medical University.

Luciferase Reporter Assay. Three identical copies of the 24 bp element flanking each allele of rs61759532 (5'-TGC TCT GGG GCG GTT AGC AAC TTC-3' for the $C$ allele and 5'-TGC TCT GGG GTG GTT AGC AAC TTC-3' for the $T$ allele) were subcloned into the luciferase vector, pGL4.26 (luc2/minP/Hydro), between the XhoI and BglII sites upstream of the minimal promoter for the firefly luciferase gene, to test the enhancing activity of the inserts (Supplementary Figure 5). The firefly luciferase vector $(1 \mu \mathrm{g})$ and normalizing Renilla luciferase vector (500 ng) were co-transfected into THP1 cells for 2 days using Lipofectamine 3000 (Thermo-Fisher Scientific). Luciferase activity was measured in five independent biological replicates using the Dual-Luciferase Reporter Assay Kit (Promega) according to the manufacturer's instructions. Relative fold-change in firefly luciferase activity was normalized by 
both transfection efficiency, based on Renilla luciferase activity, and minimal luciferase activity from the pGL4.26 vector without inserts.

EMSA. EBV-transformed B or THP1 cells were grown in RPMI 1640 medium including $10 \%$ fetal bovine serum and 1\% penicillin/streptomycin. EMSA probes were constructed by annealing biotin-conjugated 30 -residue oligonucleotide sequences flanking rs61753158: 5'-biotin-ACC TGC TCT GGG GCG GTT AGC AAC TTC CTG-3' (forward) and 5'-biotin-CAG GAA GTT GCT AAC CGC CCC AGA GCA GGT-3' (reverse) for the $C$ allele; 5'-biotin-ACC TGC TCT GGG GTG GTT AGC AAC TTC CTG-3' (forward) and 5'-biotin-CAG GAA GTT GCT AAC CAC CCC AGA GCA GGT-3' (reverse) for the $T$ allele. EMSA was performed using the LightShift Chemiluminescent EMSA Kit (Thermo-Fisher Scientific) according to the manufacturer's instructions. Briefly, a nuclear extract $(10 \mu \mathrm{g})$ of EBV-transformed B or THP1 cells was incubated with EMSA probes ( $20 \mathrm{fmol})$ for $30 \mathrm{~min}$ at room temperature in a final volume of $15 \mu \mathrm{l}$ with $1 \times$ EMSA binding buffer, after pre-incubation with a non-specific competitor poly $(\mathrm{dI}-\mathrm{dC})$ and 0 or $4 \mathrm{pmol}$ of a specific, non-conjugated competitor. DNA-protein complexes were separated on $6 \%$ nondenaturing polyacrylamide gel.

DEPICT analysis. We used DEPICT v1 release $194^{36}$ to prioritize genes and tissues and cells implicated by our genome-wide association meta-analysis results. All of the genetic variants with $\mathrm{P}<5 \times 10^{-8}$ were included. Input variants were clumped in DEPICT, using default $500 \mathrm{~kb}$ flanking regions with an LD cutoff of $\mathrm{r}^{2}$ ? $>0.1$, based on 1KGP East Asians data, yielding 1,521 autosomal loci. We applied a threshold of 
false discovery rate $(F D R) \leq 0.01$ to declare significant gene and tissue/cell enrichment.

TWAS analysis. We performed a transcriptome-wide association analysis using meta-analysis summary statistics in FUSION ${ }^{33}$ to infer gene expression changes in SLE. The training data sets for imputing gene expression were generated using eQTLs from 105 Japanese individuals in six different cell types: B cells, CD4+ ${ }^{+}$cells, $\mathrm{CD}^{+} \mathrm{T}$ cells, monocytes, natural killer (NK) cells, and peripheral blood cells ${ }^{34}$. We defined a significance threshold at Benjamini-Hochberg FDR of 0.05 to correct for multiple testing of each cell type.

\section{Gene mapping using Functional Mapping and Annotation of Genome-Wide}

Association Studies (FUMA). Potentially disease-causing genes at each SLE locus were mapped using FUMA v1.3.637 with three mapping strategies: physical, eQTL, and chromatin interaction mapping. Briefly, genes within a $10 \mathrm{~kb}$ window from lead and proxy variants ( $\mathrm{r}^{2} \geq 0.6$ in the $1 \mathrm{KGP}$ East Asians) were selected by physical mapping. eQTL mapping identified genes that were potentially cis-regulated by SLE variants within $\leq 1 \mathrm{Mb}$ distance from lead variants, using known eQTL variants with FDR-corrected $\mathrm{P}$ values $<0.05$ in immune-related cell data in eQTL databases (Supplementary Table 27). Variants were strictly filtered using combined annotation dependent depletion (CADD) $\operatorname{scores}^{63}$ ( $\left.\geq 12.37\right)$, maximum RegulomeDB $\operatorname{scores}^{64}(\leq 7)$, and maximum 15 -core chromatin state ${ }^{65}(\leq 7$; open chromatin) in any blood cell type, and FANTOM5 ${ }^{66,67}$ promoter and enhancer regions. Chromatin 
interaction mapping was used to search for genes whose promoters had chromatin interactions with cell type-specific enhancers containing SLE variants (CADD score $\geq$ 12.37, RegulomeDB score $\leq 7$ ), using Hi-C data from EBV-transformed B cells and spleen (GSE87112) ${ }^{68}$, and annotation of promoter and enhancer regions in various blood cell types and spleen ${ }^{69}$.

MAGMA-based gene prioritization. MAGMA v1.0735 was deployed to calculate gene-level disease association $\mathrm{P}$ values from variant-level association summary statistics within genes using a variant-wide mean model with data from the $1 \mathrm{KGP}$ East Asian reference panel.

Heritability estimation by LD score regression. Overall SLE heritability $h^{2}$ explained by genome-wide variants was estimated using the LD score regression model $^{21}$ with LD scores ${ }^{19}$ from 1KGP East Asian descendants, based on an SLE population prevalence of $0.03 \%$ in East Asian populations ${ }^{9}$. SLE heritability estimate was further partitioned according to known and novel SLE loci and transcription factor binding sites (TFBSs), to assess whether variants within the selected annotations explained significantly more SLE heritability using stratified LD score regression ${ }^{70}$. The boundary of each SLE locus was arbitrarily defined as $\pm 500 \mathrm{~kb}$ flanking a lead SLE-risk variant. TFBS annotations were obtained from IMPACT ${ }^{44}$. IMPACT provides 707 tissue-specific TFBSs annotation sets for 137 TFs in 23 tissues, including 58 sub-cell types. Heritability enrichment estimates for query annotations $i\left(E_{i}\right)$ were calculated as follows: 


$$
E_{i}=\frac{h_{i}^{2} / h_{G W A S}^{2}}{n_{i} / n_{G W A S}}
$$

where $h_{i}^{2}$ is heritability explained by variants within the query annotation $i, h_{G W A S}^{2}$ is the overall SLE heritability attributable to genome-wide variants, $n_{i}$ is the number of variants in the query annotation $i$, and $n_{G W A S}$ is the total number of variants analyzed. Standard errors were estimated using the block jackknife method ${ }^{70}$. Pvalues were calculated based on the $\mathrm{Z}$ score and corrected by an FDR of 0.05 . HLA variants were excluded from the TFBS-based partitioned heritability enrichment analysis.

Enrichment analysis for epigenomic features using GREGOR. Enrichment of SLE-associated variants in epigenomic regulatory features was evaluated using GREGOR $^{41}$. We utilized the Roadmap ChromHMM annotation comprising 15 chromatin states inferred by a combination of multiple histone marks in 23 immune cell types (https://egg2.wustl.edu/roadmap/web portal/). GREGOR generated 1,000 random lead variant sets with three properties for the actual SLE-risk lead variant set (distance to the nearest gene, MAF, and number of proxy variants in LD at an $r^{2}$ threshold of 0.8 in the 1KGP East Asian populations), to find a null distribution for the number of random lead variants in the annotation of interest, accounting for proxy variants. Enrichment $P$ values were derived from the upper tail for the number of actual lead SLE-risk variants in the query annotation in the null distribution, using saddle point approximation and FDR correction at 5\%. 
medRxiv preprint doi: https://doi.org/10.1101/2020.08.22.20178939; this version posted August 25, 2020. The copyright holder for this preprint (which was not certified by peer review) is the author/funder, who has granted medRxiv a license to display the preprint in perpetuity.

All rights reserved. No reuse allowed without permission.

Regulatory Element Locus Intersection (RELI) analysis. The RELI algorithm ${ }^{42}$ was used to estimate the significance of intersections between the genomic coordinates of SLE loci (defined as the lead variants and their strong LD proxies with $\mathrm{r}^{2}>0.8$ in $1 \mathrm{KGP}$ East Asians) and the DNA sequences bound by a particular TF or co-factor, as determined by ChIP-seq. We used 1,544 ChIP-seq datasets as previously described ${ }^{42}$, which contains 1,536 ChIP-seq datasets for 344 human TFs in 221 cell lines and eight viral ChIP-seq datasets from EBV-infected B cells for the EBV gene products EBNA1, EBNA2 (three datasets), EBNA3C, EBNA-LP, and Zta and from HIV-infected T-cells for TAT. We identified $371 \mathrm{ChIP-seq} \mathrm{datasets} \mathrm{for} \mathrm{TFs} \mathrm{from}$ various cell types, which had been previously suggested to form super-enhancers upon EBV infection in EBV-infected and transformed B cells ${ }^{43}$. Using RELI, we computed the significance and enrichment level for each ChIP-seq dataset by comparing the observed intersections with a null distribution of intersections obtained from 2,000 simulations. For each simulation, genetic variants were randomly chosen throughout the genome, ensuring their MAFs and LD structures similar to the actual lead variants and LD proxies. We used a significance threshold of $\mathrm{P}<10^{-6}$ after Bonferroni correction and computed relative risk by dividing the observed intersections by the mean expected number of intersections.

\section{Identification and processing of public $B$ cell line chromatin} immunoprecipitation and sequencing (ChIP-seq) datasets for allelic analysis. We identified 1,078 ChIP-seq datasets from experiments performed in B cell lines in the Gene Expression Omnibus (GE0) ${ }^{71}$ using custom text searching scripts. 505 
datasets were obtained for the GM12878 cell line and 573 for non-GM12878 cell lines. Annotations were manually checked for every dataset (assay type, cell line, and assayed molecule) to ensure accuracy. Sequence Read Archive (SRA) files obtained from GE0, representing sequencing reads, were analyzed using an automated pipeline. Briefly, the pipeline first ran quality control (QC) on the FastQ files using FastQC (v0.11.8) ${ }^{72}$. If FastQC detected adapter sequences, the pipeline ran the FastQ files through Trim Galore (v0.4.2)73, a wrapper script that runs cutadapt (v1.9.1 $)^{74}$, to remove the detected adapter sequences from the reads. The quality controlled reads were then aligned to the reference human genome (hg19/GRCh37) using bowtie2 (v2.3.4.1)75, followed by sorting using samtools (v1.8.0) ${ }^{76}$ and removing duplicate reads using picard (v1.89) ${ }^{77}$. Finally, peaks were called using MACS2 (v2.1.2)62, with four "modes" using the following parameter settings: MODE1 = -g hs -q 0.01; MODE2 = -g hs -q 0.01 -broad; MODE3 = -g hs -q 0.01 -broad --nomodel --extsize 500 ; and MODE4 = -g hs -q 0.01 --broad --nomodel -extsize 1000. Peaks were merged across the four MODES using bedTools to produce a final peak set for each experiment. ENCODE blacklist regions ${ }^{78}$ were removed from the peak sets using the hg19-blacklist.v2.bed.gz file available at https://github.com/Boyle-Lab/Blacklist/blob/master/lists/hg19-

blacklist.v2.bed.gz. 93 datasets failed at the download, alignment, or peak calling steps, yielding a total of 985 ChIP-seq peak sets in .BED format for subsequent analysis. 
medRxiv preprint doi: https://doi.org/10.1101/2020.08.22.20178939; this version posted August 25, 2020. The copyright holder for this preprint (which was not certified by peer review) is the author/funder, who has granted medRxiv a license to display the preprint in perpetuity.

All rights reserved. No reuse allowed without permission.

Allele-dependent ChIP-seq data analysis. We used whole genome sequencing data from 1KGP phase 3 to identify heterozygous genetic variants in B cell lines. Starting with variant call files produced by the 1KGP, heterozygous variants were identified for each subject. For the GM12878 cell line, we used genotyping information obtained from Illumina 0MNI-5 arrays. Genotypes were called using the Gentrain2 algorithm within Illumina Genome Studio. Quality control was performed as previously described ${ }^{79}$. Quality control data cleaning was performed in the context of a larger batch of non-disease controls to allow for data quality assessment. Briefly, all cell lines had call rates $>99 \%$; only variants with MAF $>0.01$ were included; and all variants with $\mathrm{P}_{\mathrm{HWE}}>10^{-4}$ were included. We performed genome-wide imputation using overlapping $150 \mathrm{~kb}$ sections of the genome with IMPUTE $2^{55}$ and 1 KGP phase 3 (June 2014), and then filtered imputed variants with probability $<0.9$ or imputation quality $<0.5$ in addition to the same criteria described above for typed markers. Regions of the genome with abnormal chromosome counts (i.e., regions that did not have two chromosomes) were removed from consideration using the cnvPartitionsoftware package (Illumina Genome Studio) with default parameter settings, due to their potential effects on allelic read imbalances.

Next, as a final quality control step, the identity of cell lines, as annotated in GE0, was confirmed by assessing read counts at variants identified as heterozygous. For each dataset in each cell line, we examined all heterozygotes with at least five sequencing reads. We then calculated the fraction of these variants with exactly zero reads on the weak allele (i.e., the allele with fewer mapped sequencing reads). Zero weak allele reads is a hallmark of a mis-annotated cell line, since a variant that is 
thought to be a heterozygote but in reality is a homozygote will always exhibit zero weak allele reads. Any dataset $\geq 45 \%$ "zero weak allele read heterozygotes" was flagged as a mis-annotation by the original producers of the dataset and removed from downstream analyses. This cutoff was chosen based on comparisons between purposely matched and mis-matched genotyping array/ChIP-seq experiment pairs. In total, 482 datasets were used for allelic analyses.

To identify possible mechanisms underlying identified allelic variants, we applied the MARIO method ${ }^{42}$ to the ChIP-seq dataset collection. Briefly, MARIO identifies common genetic variants that are (1) heterozygous in the assayed cell line and (2) located within a peak in a given ChIP-seq dataset. It then examines the sequencing reads that map to each heterozygote in each peak for imbalance between the two alleles. MARIO Allelic Reproducibility Score (ARS) values $>0.4$ were considered allelic, following our previous study ${ }^{42}$. We also used an additional filter to ensure consistency across ChIP-seq datasets. Specifically, for each variant/regulatory molecule pair, we calculated the fraction of ChIP-seq datasets that shared the same preferred allele. For example, if a particular variant was a heterozygote located inside an H3K27ac ChIP-seq peak in 10 datasets, and five of them preferred the G allele (with ARS value > 0.4), this value would be 0.5 . We only included variant/regulatory molecule pairs where this value was $\geq 0.3$.

In-silico mutagenesis to pinpoint causal variants and genes. To explore the mutation effect and then to prioritize the causal variants and potential regulatory mechanisms for SLE genetic associations, we applied Mutation effect prediction on 
medRxiv preprint doi: https://doi.org/10.1101/2020.08.22.20178939; this version posted August 25, 2020. The copyright holder for this preprint (which was not certified by peer review) is the author/funder, who has granted medRxiv a license to display the preprint in perpetuity.

All rights reserved. No reuse allowed without permission.

ncRNA transcription (MENTR) ${ }^{45}$ analysis in significant summary-level associations from current meta-analysis. MENTR learns cell-type-specific transcription probability of promoters and enhancers surrounding transcription start sites (TSSs) using FANTOM5 Cap Analysis of Gene Expression (CAGE) ${ }^{80}$ from 347 types of samples comprising a variety of primary cells and tissues, and then predicts mutation effects on transcripts by in silico mutagenesis for certain variants based on the degree of probability change. In order to investigate mutation effects for 9,506 significant non-HLA SNPs $\left(\mathrm{P}<5 \times 10^{-8}\right)$ on transcription, we searched for genes whose TSS are less than $100 \mathrm{~kb}$ from any significant non-HLA SNP and found 350,410 variant-gene pairs. We then evaluated the mutation effect for these 350,410 variantgene pairs in MENTR. We identified 2,383 top variant-gene-cell sets that had robust mutation effects at mutation effect $>0.1$, which shows high accuracy of prediction ${ }^{45}$. Among these top variant-gene-cell sets, we eventually restricted the results to the 71 sets that contain lead variants, proxies of strong LD with lead variants $\left(r^{2}>0.8\right.$ in the combined genotypes of $1 \mathrm{KGP}$ phase 3 v5 East Asians and whole genome sequencing data for 7,472 Japanese unrelated individual), and immune cells.

\section{Genetic correlation between SLE and diseases/traits using LD score regression.}

We calculated genetic correlations between 98 traits $\left(39\right.$ diseases ${ }^{17}$ and 59 quantitative traits $^{81}$ ) and SLE by using LD score regression ${ }^{48}$. SNPs were restricted to HapMap3, because these are well-imputed in most studies. The $H L A$ region was excluded because of its complex LD structure. LD scores of the 1KGP East Asians 
medRxiv preprint doi: https://doi.org/10.1101/2020.08.22.20178939; this version posted August 25, 2020. The copyright holder for this preprint (which was not certified by peer review) is the author/funder, who has granted medRxiv a license to display the preprint in perpetuity.

All rights reserved. No reuse allowed without permission.

were used to estimate genetic correlations. Significance was defined as Benjamini-

Hochberg FDR of 0.05 . 
medRxiv preprint doi: https://doi.org/10.1101/2020.08.22.20178939; this version posted August 25, 2020. The copyright holder for this preprint (which was not certified by peer review) is the author/funder, who has granted medRxiv a license to display the preprint in perpetuity.

\section{Acknowledgements}

We acknowledged the participants in this study. We appreciate the contribution of Japanese Research Committee on Idiopathic Osteonecrosis of the Femoral Head. We appreciate all contributors to BioBank Japan. Details are included in supplementary material. This research was supported by General Program (81872516, 81573033, 81872527, 81830019, 81421001), Young Program (81803117), Exchange Program (81881340424), and Science Fund for Creative Research Groups (31630021) of National Natural Science Foundation of China (NSFC), Distinguished Young Scholar of Provincial Natural Science Foundation of Anhui (1808085J08), National Program on Key Basic Research Project of China (973 Program) (2014CB541901), Science Foundation of Ministry of Education of China (213018A), Program for New Century Excellent Talents in University of Ministry of Education of China (NCET-12-0600), The Bio \& Medical Technology Development Program of the National Research Foundation, funded by the Ministry of Science \& ICT of the Republic of Korea (NRF2017M3A9B4050355 to S.C.B.), Basic Science Research Program through the National Research Foundation of Korea funded by the Ministry of Science, ICT and Future Planning (2015R1C1A1A02036527 and 2017R1E1A1A01076388 to K. Kim), National BioBank of Korea, the Centers for Disease Control and Prevention, Republic of Korea (KBN-2018-031 to S.S.L.), Center for Genome Science, Korea National Institute of Health, Republic of Korea (4845-301, 3000-3031 to M.Y.H., K. Yoon and B.J.K.), Japan Agency for Medical Research and Development (AMED) and the BioBank Japan project supported by the Ministry of Education, Culture, Sports, Sciences and Technology of the Japanese Government and AMED under grant 
medRxiv preprint doi: https://doi.org/10.1101/2020.08.22.20178939; this version posted August 25, 2020. The copyright holder for this preprint (which was not certified by peer review) is the author/funder, who has granted medRxiv a license to display the preprint in perpetuity.

All rights reserved. No reuse allowed without permission.

numbers (17km0305002 and 18km0605001), Grant of Japan Orthopaedics and

Traumatology Research Foundation, lnc, (No.350 to Y.Sakamoto), RIKEN Junior

Research Associate Program (to H.S.), US NIH grants (AI024717, AI130830,

AI148276, HG172111 and AR070549 to J.B.H.), US Department of Veterans Affairs (BX001834 to J.B.H.), and Center for Pediatric Genomics Award and CCRF Endowed Scholar Award of Cincinnati Children's Hospital (to M.T.W.). We appreciate the generous gift of pGL4.26 vector from Prof. Joon Kim at Graduate School of Medical Science Engineering, KAIST, Daejeon, South Korea.

\section{Author Contributions}

X.Y., K. Kim and H.S. contributed equally to this work, and either has the right to list himself first in bibliographic documents. S.C.B., Y.C., C.T., X. Zhang, X.Y., K. Kim and H.S. conceived the study design. S.C.B., Y.C, X. Zhang, S.Y., K. Kim and C.T. acquainted the financial support. X.Y., K. Kim, H.S., C.T., Y.C. and S.C.B. wrote the manuscript. X.Y., K. Kim, H.S., E.H., X. Zheng, V.L. and Y.W. conducted all of the analyses with the help of J.B.H., L.C.K., M.T.W., S.P., S.E., H.S., K.T., N.O., M.K., K.I. and C.Terao. K. Kim, S.Y.B, L.W., L.L., R.X.L., Y. Sheng, M.Y.H., W.L., K. Yoon, M.C., H.H., M.W., Y. Tang, H.D., C.L., C.S., W.F., K.L., B.J.K., H.S.L., S.C.B., S.H., Y.Sakamoto, N.Sugano, M.M., D.T., K.Karino, T.Miyamura, J.N., G.M., T.Kuroda, H.N., T.Miyamoto, T.T., Y.Kawaguchi, K.A., Y.Tada, K.Yamaji, M.S., T.A., A.S., T.Sumida, Y.Okada, K.Matsuda, K.Matsuo, Y.Kochi, T.Seki, Y.Tanaka, T.Kubo, R.H., T.Yoshioka, M.Y., T.Kabata, Y.A., Y.Ohta, T.O., Y.N., A.K., Y.Y., K.Ohzono, K.Yamamoto, K.Ohmura, T.Yamamoto and S.I. generated genetic data. L.L. and H.H. contributed to ATAC-seq experiment. S.J. and T.H.K. performed luciferase 
medRxiv preprint doi: https://doi.org/10.1101/2020.08.22.20178939; this version posted August 25, 2020. The copyright holder for this preprint (which was not certified by peer review) is the author/funder, who has granted medRxiv a license to display the preprint in perpetuity.

reporter assays and EMSAs. S.Y.B., Y.C.K., W.T.C., S.S.L., S.C.S., Y.M.K., D.Y., C.H.S.,

Y.B.P., J.Y.C., Y.P., G.Y.A., J.M.S., Y.K.L., W.Y., S.Y., B.J.K., N. Shen, H.S.L., X. Zhang, C.T.

and S.C.B. managed the cohort data.

\section{Competing Interests}

The authors declare no competing interests.

\section{References}

1. Akizuki, S. et al. PLD4 is a genetic determinant to systemic lupus erythematosus and involved in murine autoimmune phenotypes. Ann Rheum Dis 78, 509-518 (2019).

2. Kim, K. et al. The HLA-DR $\beta 1$ amino acid positions 11-13-26 explain the majority of SLE-MHC associations. Nat Commun 5, 5902 (2014).

3. Gateva, V. et al. A large-scale replication study identifies TNIP1, PRDM1, JAZF1, UHRF1BP1 and IL10 as risk loci for systemic lupus erythematosus. Nat Genet 41, 1228-33 (2009).

4. Han, J.W. et al. Genome-wide association study in a Chinese Han population identifies nine new susceptibility loci for systemic lupus erythematosus. Nat Genet 41, 1234-7 (2009).

5. Morris, D.L. et al. Genome-wide association meta-analysis in Chinese and European individuals identifies ten new loci associated with systemic lupus erythematosus. Nat Genet 48, 940-946 (2016).

6. Sun, C. et al. High-density genotyping of immune-related loci identifies new SLE risk variants in individuals with Asian ancestry. Nat Genet 48, 323-30 (2016).

7. Cunninghame Graham, D.S. et al. Association of NCF2, IKZF1, IRF8, IFIH1, and TYK2 with systemic lupus erythematosus. PLoS Genet 7, e1002341 (2011).

8. Okada, Y. et al. A genome-wide association study identified AFF1 as a susceptibility locus for systemic lupus eyrthematosus in Japanese. PLoS Genet 8, e1002455 (2012).

9. Carter, E.E., Barr, S.G. \& Clarke, A.E. The global burden of SLE: prevalence, health disparities and socioeconomic impact. Nat Rev Rheumatol 12, 605-20 (2016).

10. Guerra, S.G., Vyse, T.J. \& Cunninghame Graham, D.S. The genetics of lupus: a functional perspective. Arthritis Res Ther 14, 211 (2012).

11. Catalina, M.D., Owen, K.A., Labonte, A.C., Grammer, A.C. \& Lipsky, P.E. The pathogenesis of systemic lupus erythematosus: Harnessing big data to understand the molecular basis of lupus. J Autoimmun 110, 102359 (2020). 
12. Bentham, J. et al. Genetic association analyses implicate aberrant regulation of innate and adaptive immunity genes in the pathogenesis of systemic lupus erythematosus. Nat Genet 47, 1457-1464 (2015).

13. Buniello, A. et al. The NHGRI-EBI GWAS Catalog of published genome-wide association studies, targeted arrays and summary statistics 2019. Nucleic Acids Res 47, D1005-d1012 (2019).

14. Sirugo, G., Williams, S.M. \& Tishkoff, S.A. The Missing Diversity in Human Genetic Studies. Cell 177, 26-31 (2019).

15. Danchenko, N., Satia, J.A. \& Anthony, M.S. Epidemiology of systemic lupus erythematosus: a comparison of worldwide disease burden. Lupus 15, 30818 (2006).

16. Morais, S.A. \& Isenberg, D.A. A study of the influence of ethnicity on serology and clinical features in lupus. Lupus 26, 17-26 (2017).

17. Ishigaki, K. et al. Large-scale genome-wide association study in a Japanese population identifies novel susceptibility loci across different diseases. Nat Genet (2020).

18. Yang, W. et al. Genome-wide association study in Asian populations identifies variants in ETS1 and WDFY4 associated with systemic lupus erythematosus. PLoS Genet 6, e1000841 (2010).

19. Auton, A. et al. A global reference for human genetic variation. Nature 526, 68-74 (2015).

20. Kim, Y., Kim, B. \& Han, B. The Korean Reference Genome project: construction of the refer- ence panel for imputation analysis. Presented at the 61th Annual Meeting of The American Society of Human Genetics, October 6, 2015, Baltimore, MD) Abstract/Program \#3163F(2015).

21. Bulik-Sullivan, B.K. et al. LD Score regression distinguishes confounding from polygenicity in genome-wide association studies. Nat Genet 47, 291-5 (2015).

22. Yang, J. et al. Conditional and joint multiple-SNP analysis of GWAS summary statistics identifies additional variants influencing complex traits. Nat Genet 44, 369-75, s1-3 (2012).

23. Wen, L. et al. Exome-wide association study identifies four novel loci for systemic lupus erythematosus in Han Chinese population. Ann Rheum Dis 77, 417 (2018).

24. Zhang, Y.et al. Meta-analysis of GWAS on two Chinese populations followed by replication identifies novel genetic variants on the $\mathrm{X}$ chromosome associated with systemic lupus erythematosus. Hum Mol Genet 24, 274-84 (2015).

25. Armstrong, D.L., Eisenstein, M., Zidovetzki, R. \& Jacob, C.0. Systemic lupus erythematosus-associated neutrophil cytosolic factor 2 mutation affects the structure of NADPH oxidase complex. J Biol Chem 290, 12595-602 (2015).

26. Kim-Howard, X. et al. Allelic heterogeneity in NCF2 associated with systemic lupus erythematosus (SLE) susceptibility across four ethnic populations. Hum Mol Genet 23, 1656-68 (2014).

27. Sannigrahi, M.K., Srinivas, C.S., Deokate, N. \& Rakshit, S. The strong propensity of Cadherin-23 for aggregation inhibits cell migration. Mol Oncol 13, 1092-1109 (2019). 
28. Morimoto, K. et al. LRRK1 is critical in the regulation of B-cell responses and CARMA1-dependent NF-кB activation. Sci Rep 6, 25738 (2016).

29. Benner, C. et al. FINEMAP: efficient variable selection using summary data from genome-wide association studies. Bioinformatics 32, 1493-501 (2016).

30. Pasula, S. et al. Role of Systemic Lupus Erythematosus Risk Variants With Opposing Functional Effects as a Driver of Hypomorphic Expression of TNIP1 and Other Genes Within a Three-Dimensional Chromatin Network. Arthritis Rheumatol 72, 780-790 (2020).

31. Chen, P.W., Luo, R., Jian, X. \& Randazzo, P.A. The Arf6 GTPase-activating proteins ARAP2 and ACAP1 define distinct endosomal compartments that regulate integrin $\alpha 5 \beta 1$ traffic. J Biol Chem 289, 30237-48 (2014).

32. Human genomics. The Genotype-Tissue Expression (GTEx) pilot analysis: multitissue gene regulation in humans. Science 348, 648-60 (2015).

33. Gusev, A. et al. Integrative approaches for large-scale transcriptome-wide association studies. Nat Genet 48, 245-52 (2016).

34. Ishigaki, K. et al. Polygenic burdens on cell-specific pathways underlie the risk of rheumatoid arthritis. Nat Genet 49, 1120-1125 (2017).

35. de Leeuw, C.A., Mooij, J.M., Heskes, T. \& Posthuma, D. MAGMA: generalized gene-set analysis of GWAS data. PLoS Comput Biol 11, e1004219 (2015).

36. Pers, T.H. et al. Biological interpretation of genome-wide association studies using predicted gene functions. Nat Commun 6, 5890 (2015).

37. Watanabe, K., Taskesen, E., van Bochoven, A. \& Posthuma, D. Functional mapping and annotation of genetic associations with FUMA. Nat Commun 8, 1826 (2017).

38. Suzuki, N., Ichino, M., Mihara, S., Kaneko, S. \& Sakane, T. Inhibition of Fas/Fas ligand-mediated apoptotic cell death of lymphocytes in vitro by circulating anti-Fas ligand autoantibodies in patients with systemic lupus erythematosus. Arthritis Rheum 41, 344-53 (1998).

39. Deng, Y. et al. Decreased SMG7 expression associates with lupus-risk variants and elevated antinuclear antibody production. Ann Rheum Dis 75, 2007-2013 (2016).

40. Zhang, W. et al. Extended haplotype association study in Crohn's disease identifies a novel, Ashkenazi Jewish-specific missense mutation in the NF- $\kappa \mathrm{B}$ pathway gene, HEATR3. Genes Immun 14, 310-6 (2013).

41. Schmidt, E.M. et al. GREGOR: evaluating global enrichment of trait-associated variants in epigenomic features using a systematic, data-driven approach. Bioinformatics 31, 2601-6 (2015).

42. Harley, J.B. et al. Transcription factors operate across disease loci, with EBNA2 implicated in autoimmunity. Nat Genet 50, 699-707 (2018).

43. Zhou, H. et al. Epstein-Barr virus oncoprotein super-enhancers control B cell growth. Cell Host Microbe 17, 205-16 (2015).

44. Amariuta, T. et al. IMPACT: Genomic Annotation of Cell-State-Specific Regulatory Elements Inferred from the Epigenome of Bound Transcription Factors. Am J Hum Genet 104, 879-895 (2019).

45. Koido, M. et al. Predicting cell-type-specific non-coding RNA transcription from genome sequence. Preprint at 
medRxiv preprint doi: https://doi.org/10.1101/2020.08.22.20178939; this version posted August 25, 2020. The copyright holder for this preprint

(which was not certified by peer review) is the author/funder, who has granted medRxiv a license to display the preprint in perpetuity.

All rights reserved. No reuse allowed without permission.

https://www.biorxiv.org/content/10.1101/2020.03.29.011205v2.full. (2020).

46. Clark, D.N. et al. Molecular effects of autoimmune-risk promoter polymorphisms on expression, exon choice, and translational efficiency of interferon regulatory factor 5. J Interferon Cytokine Res 34, 354-65 (2014).

47. Pamuk, 0.N. et al. BLK pathway-associated rs13277113 GA genotype is more frequent in SLE patients and associated with low gene expression and increased flares. Clin Rheumatol 36, 103-109 (2017).

48. Bulik-Sullivan, B. et al. An atlas of genetic correlations across human diseases and traits. Nat Genet 47, 1236-41 (2015).

49. Kwon, O.C. et al. Predicting eventual development of lupus nephritis at the time of diagnosis of systemic lupus erythematosus. Semin Arthritis Rheum 48, 462-466 (2018).

50. Moon, S. et al. The Korea Biobank Array: Design and Identification of Coding Variants Associated with Blood Biochemical Traits. Sci Rep 9, 1382 (2019).

51. Hochberg, M.C. Updating the American College of Rheumatology revised criteria for the classification of systemic lupus erythematosus. Arthritis Rheum 40, 1725 (1997).

52. O'Connell, J. et al. A general approach for haplotype phasing across the full spectrum of relatedness. PLoS Genet 10, e1004234 (2014).

53. Loh, P.R. et al. Reference-based phasing using the Haplotype Reference Consortium panel. Nat Genet 48, 1443-1448 (2016).

54. Bycroft, C. et al. The UK Biobank resource with deep phenotyping and genomic data. Nature 562, 203-209 (2018).

55. Howie, B.N., Donnelly, P. \& Marchini, J. A flexible and accurate genotype imputation method for the next generation of genome-wide association studies. PLoS Genet 5, e1000529 (2009).

56. Das, S. et al. Next-generation genotype imputation service and methods. Nat Genet 48, 1284-1287 (2016).

57. Purcell, S. et al. PLINK: a tool set for whole-genome association and population-based linkage analyses. Am J Hum Genet 81, 559-75 (2007).

58. Marchini, J. \& Howie, B. Genotype imputation for genome-wide association studies. Nat Rev Genet 11, 499-511 (2010).

59. Cook, J.P., Mahajan, A. \& Morris, A.P. Guidance for the utility of linear models in meta-analysis of genetic association studies of binary phenotypes. Eur J Hum Genet 25, 240-245 (2017).

60. Willer, C.J., Li, Y. \& Abecasis, G.R. METAL: fast and efficient meta-analysis of genomewide association scans. Bioinformatics 26, 2190-1 (2010).

61. Buenrostro, J.D., Giresi, P.G., Zaba, L.C., Chang, H.Y. \& Greenleaf, W.J. Transposition of native chromatin for fast and sensitive epigenomic profiling of open chromatin, DNA-binding proteins and nucleosome position. Nat Methods 10, 1213-8 (2013).

62. Zhang, Y. et al. Model-based analysis of ChIP-Seq (MACS). Genome Biol 9, R137 (2008).

63. Kircher, M. et al. A general framework for estimating the relative pathogenicity of human genetic variants. Nat Genet 46, 310-5 (2014). 
64. Boyle, A.P. et al. Annotation of functional variation in personal genomes using RegulomeDB. Genome Res 22, 1790-7 (2012).

65. Ernst, J. \& Kellis, M. ChromHMM: automating chromatin-state discovery and characterization. Nat Methods 9, 215-6 (2012).

66. Andersson, R. et al. An atlas of active enhancers across human cell types and tissues. Nature 507, 455-461 (2014).

67. Forrest, A.R. et al. A promoter-level mammalian expression atlas. Nature 507, 462-70 (2014).

68. Schmitt, A.D. et al. A Compendium of Chromatin Contact Maps Reveals Spatially Active Regions in the Human Genome. Cell Rep 17, 2042-2059 (2016).

69. Kundaje, A. et al. Integrative analysis of 111 reference human epigenomes. Nature 518, 317-30 (2015).

70. Finucane, H.K. et al. Partitioning heritability by functional annotation using genome-wide association summary statistics. Nat Genet 47, 1228-35 (2015).

71. Barrett, T. et al. NCBI GEO: archive for functional genomics data sets--update. Nucleic Acids Res 41, D991-5 (2013).

72. Kalita, C.A. et al. QuASAR-MPRA: accurate allele-specific analysis for massively parallel reporter assays. Bioinformatics 34, 787-794 (2018).

73. Goodwin, S., McPherson, J.D. \& McCombie, W.R. Coming of age: ten years of next-generation sequencing technologies. Nat Rev Genet 17, 333-51 (2016).

74. Bentley, D.R. et al. Accurate whole human genome sequencing using reversible terminator chemistry. Nature 456, 53-9 (2008).

75. Langmead, B. \& Salzberg, S.L. Fast gapped-read alignment with Bowtie 2. Nat Methods 9, 357-9 (2012).

76. Li, H. et al. The Sequence Alignment/Map format and SAMtools. Bioinformatics 25, 2078-9 (2009).

77. Vaughn, S.E. et al. Lupus risk variants in the PXK locus alter B-cell receptor internalization. Front Genet 5, 450 (2014).

78. Amemiya, H.M., Kundaje, A. \& Boyle, A.P. The ENCODE Blacklist: Identification of Problematic Regions of the Genome. Sci Rep 9, 9354 (2019).

79. Kottyan, L.C. et al. Genome-wide association analysis of eosinophilic esophagitis provides insight into the tissue specificity of this allergic disease. Nat Genet 46, 895-900 (2014).

80. Noguchi, S. et al. FANTOM5 CAGE profiles of human and mouse samples. Sci Data 4, 170112 (2017).

81. Terao, C. et al. GWAS of mosaic loss of chromosome Y highlights genetic effects on blood cell differentiation. Nat Commun 10, 4719 (2019). 
Figure Legends
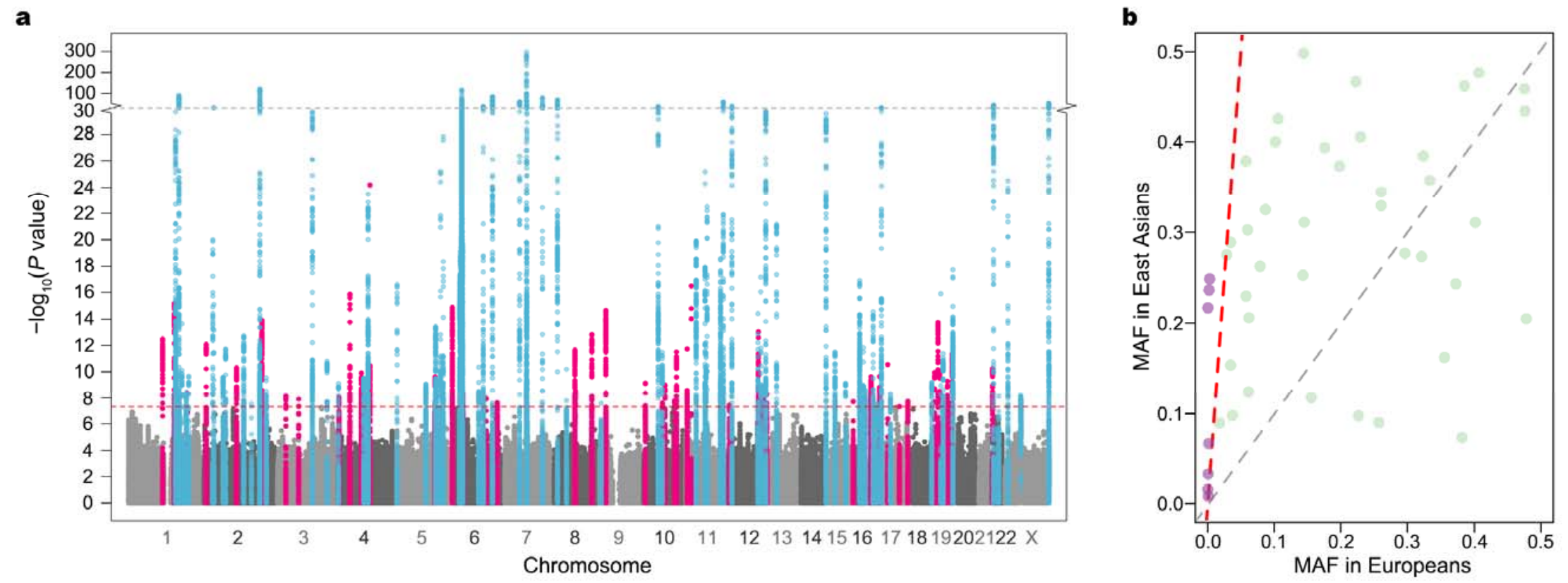

Fig. 1 | Summary of meta-analysis association results and comparison of MAFs for lead variants within the 46 novel

loci between East Asians and Europeans. a, Manhattan plot of genome-wide association meta-analysis results from 208,370

SLE case-control East Asians. Minus $\log _{10}$-transformed association $P$ values (y-axis) are plotted along chromosomal positions (x-axis). Known and novel loci are highlighted in light blue and pink, respectively. The red dashed line denotes the genomewide association significance threshold of $P=5 \times 10^{-8}$. The gray dashed line represents $P=10^{-30}$, at which the y-axis breaks. $\mathbf{b}$, 
Comparison of MAFs of lead variants within the 46 novel loci between East Asians (y-axis) and non-Finnish Europeans ( $\mathrm{x}$-axis) in the Genome Aggregation Database (gnomAD) version 3. Variants with more than ten-times higher MAFs in East Asians are colored purple above a red dashed line. 
a

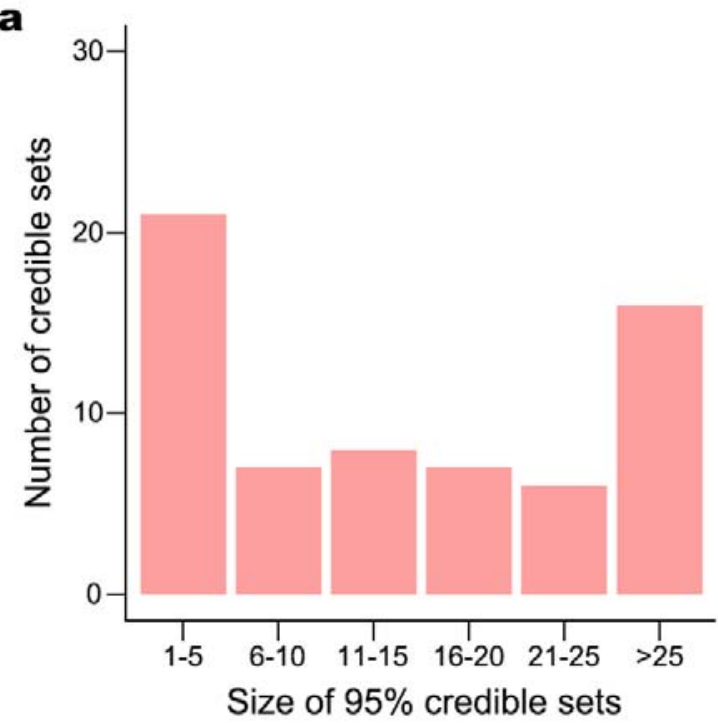

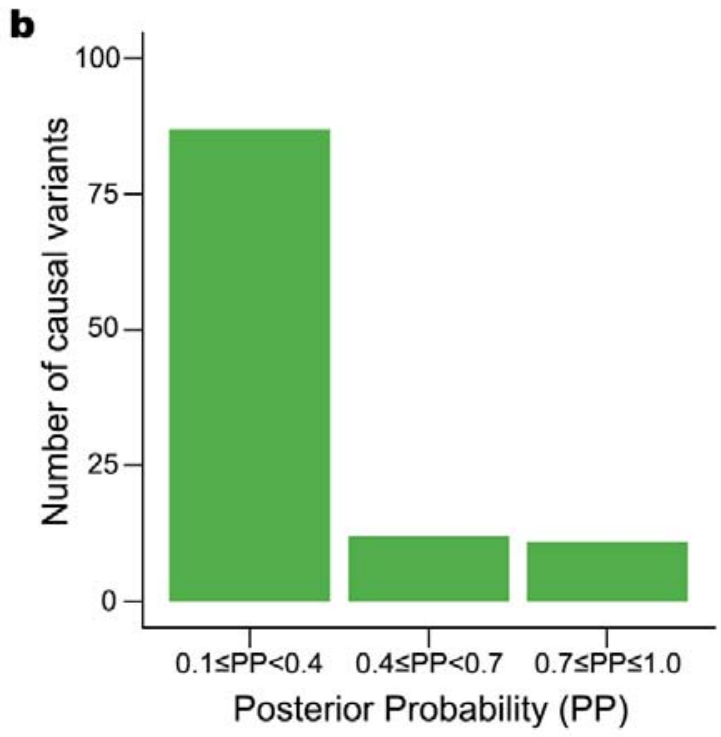

Fig. 2 | Results of statistical fine-mapping. a, Number of 95\% credible sets of putative causal variants, binned by their sizes. b, Number of potential causal variants with posterior probabilities $(\mathrm{PP}) \geq 0.1$, which are considered to be the true causal variants. 
a

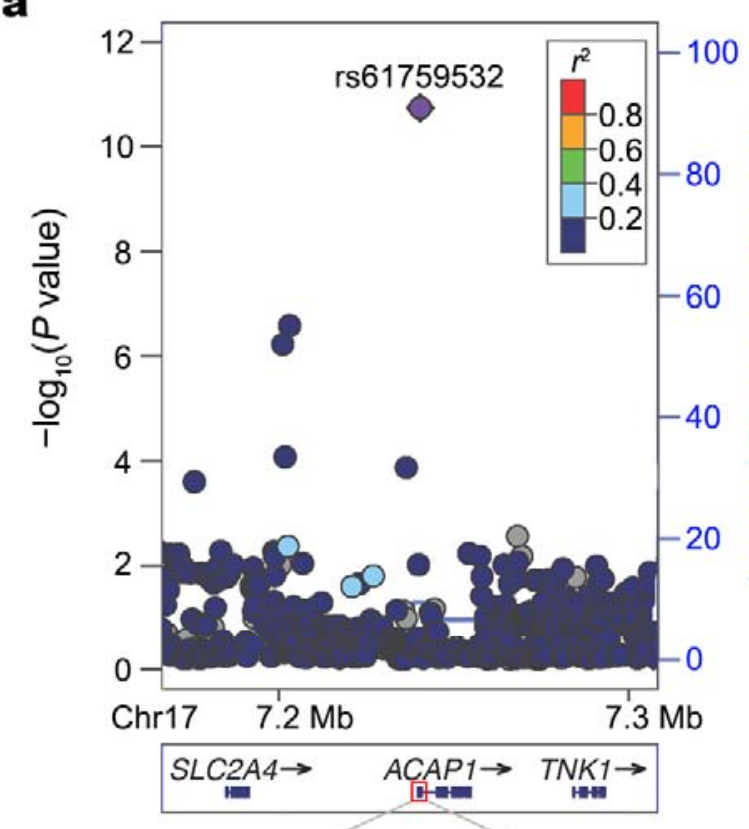

b

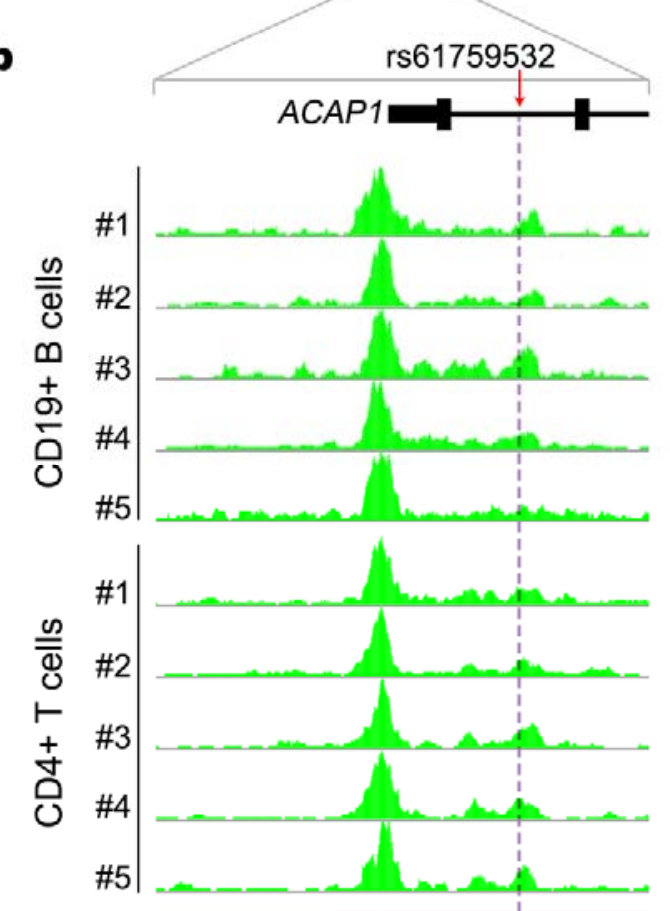

CD8.NPC

CD4.NPC CD19.PPC

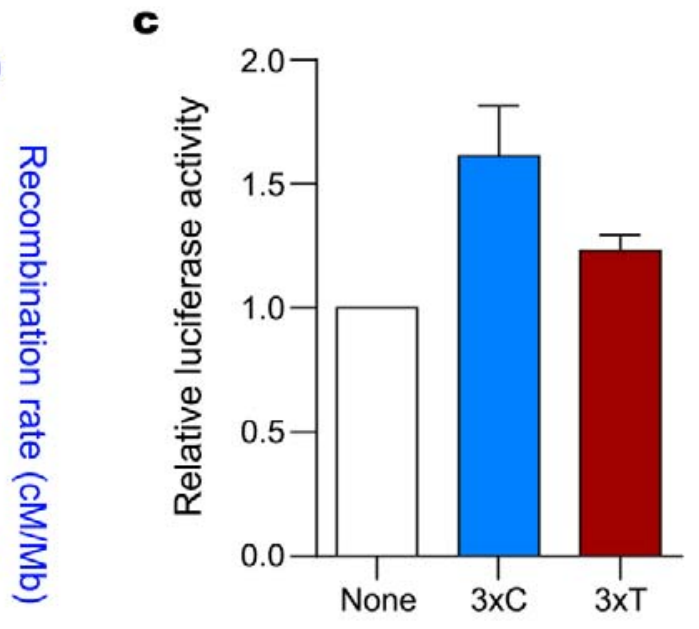

d

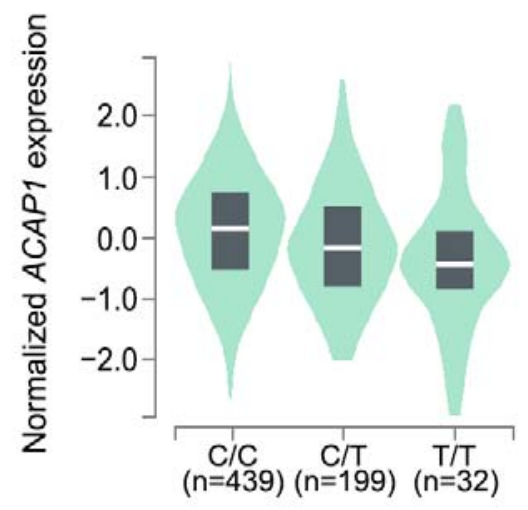

e

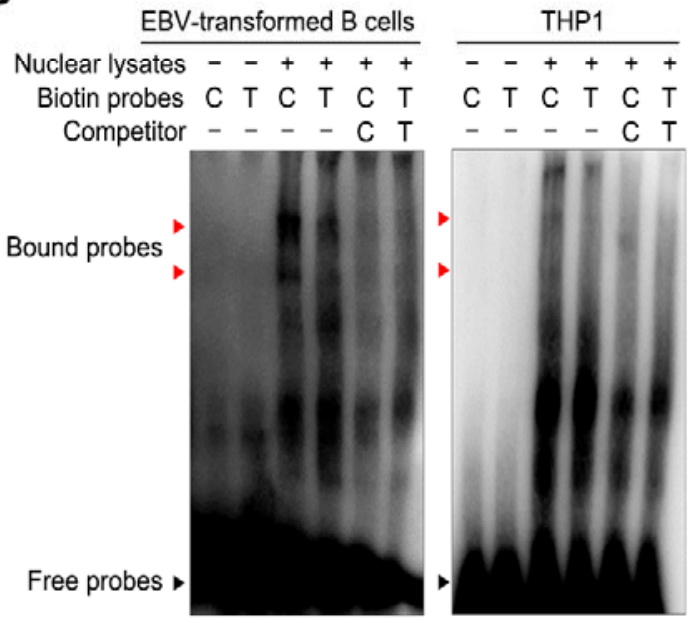

Fig. 3 | Allele-specific regulatory effect of rs61759532 on ACAP1. a, Regional association plot for the ACAP1 locus. The lead variant rs61759532 is labeled as a 
purple diamond. The LD was estimated using data from 7,021 Chinese individuals. b, Location of rs61759532 within an ATAC-seq open chromatin accessible region in CD19+ $\mathrm{B}-$ and CD4+ ${ }^{+}$-cells (green tracks) and within active ChromHMM chromatin states (bars on the bottom panel) in primary CD8 ${ }^{+}$T naive cells (CD8.NPC), T helper naive cells (CD4.NPC), and primary B cells (BLD.CD19.PPC). Chromatin states are colored red (active transcription start site), orange red (flanking active transcription start site, and yellow (enhancers).c, Allelic differentials in the enhancing activity of rs61759532 in THP1 cells. None, $3 \times$ C, and $3 \times$ T denote empty vector containing a minimal promoter, and vectors with the $C$ and $T$ allele of rs61759532, respectively. Relative luciferases activities, measured in five independent biological replicates, were significantly higher for inserts with the $C$ allele $\left(P=8.1 \times 10^{-3}\right.$; two-tailed t-test $)$. Error bars indicate standard errors of the means of five independent biological replicates. d, Association between the risk allele (T) of rs61759532 and decreased expression of ACAP1 in GTEx v8 whole blood $\left(P=1.7 \times 10^{-47}\right)$. The white line in the center of each box indicates median expression value, while the box for each genotype represents the interquartile range of $A C A C P 1$ expressions. e, Allelic differential in protein-DNA binding by rs61759532 in EMSAs. Biotin-conjugated 30-nucleotide probes flanking rs61759532 (denoted as $C$ or $T$, according the allele) were incubated with nuclear extracts (10 $\mu \mathrm{g}$ ) from EBVtransformed B cells or THP1 cells in EMSAs. Shifted bands (indicated by red arrows) had stronger intensities with biotin-conjugated $C$ allele probes than $T$ allele probes, and were not detected in the presence of excess non-conjugated probes. 
a

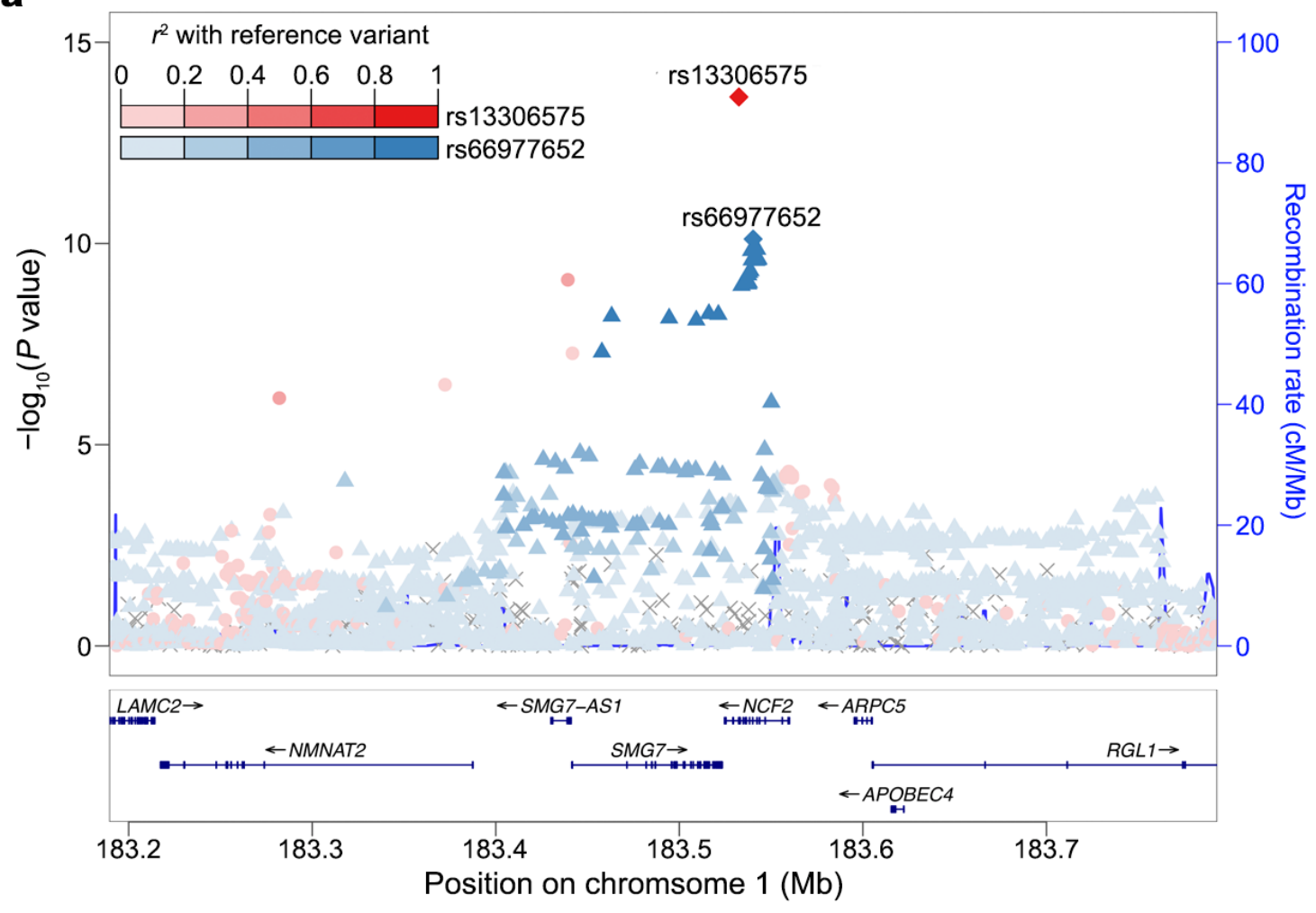

b
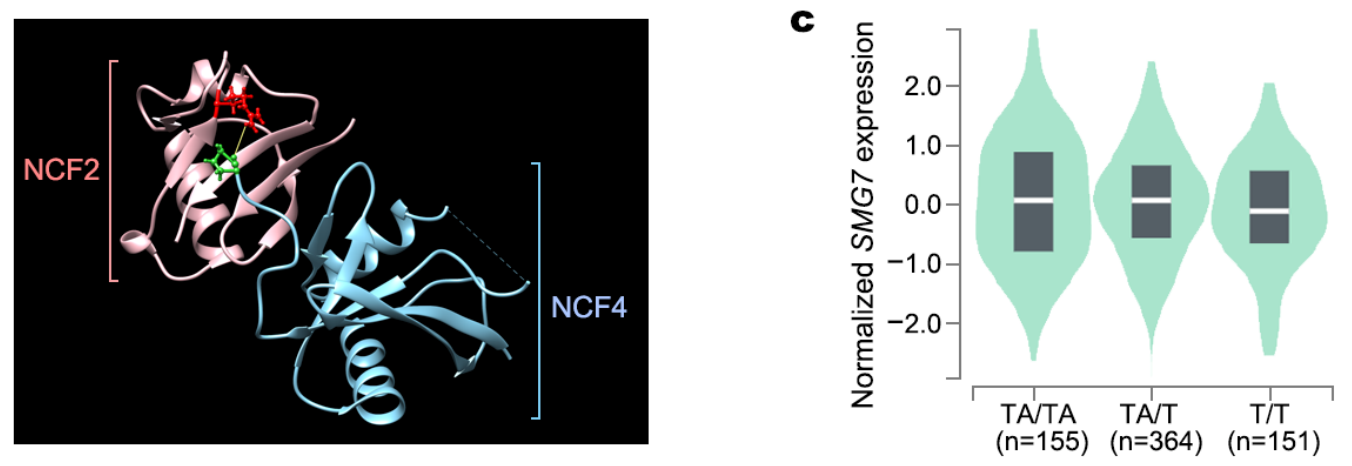

Fig. 4 | Distinct effects of the two independent association signals (rs13306575 and rs66977652) at a single locus containing NCF2 and SMG7. a, Regional association in the locus including NCF2 and SMG7. Lead and secondary index variants are indicated by diamonds. The lead variant and its LD proxies are in red, while the secondary signal index variant and its LD proxies are in blue. LD was estimated using data from 7,021 unrelated Chinese reference samples. b, Arginine at 
position 395 (red) of NCF2 (pink) is known to interact with proline at position 339 (green) of NCF4 (cyan) via a hydrogen bond (yellow) to form an NADPH oxidase complex. The substitution of arginine at position 395 to tryptophan, induced by the risk allele (A) of the lead missense variant rs13306575 (p.Arg395Trp) in NCF2, likely disrupts this interaction. c, The protective $\mathrm{T}$ allele of the secondary signal rs66977652 is associated with decreased expression levels of SMG7 in a wide range of GTEx v8 human tissues, including whole blood $\left(P=3.1 \times 10^{-15}\right)$. Sample sizes according to rs66977652 genotypes are included in parentheses on the $\mathrm{x}$-axis. The white line in the center of each box indicates median expression value, while the box for each genotype represents the interquartile range of SMG7 expressions. 

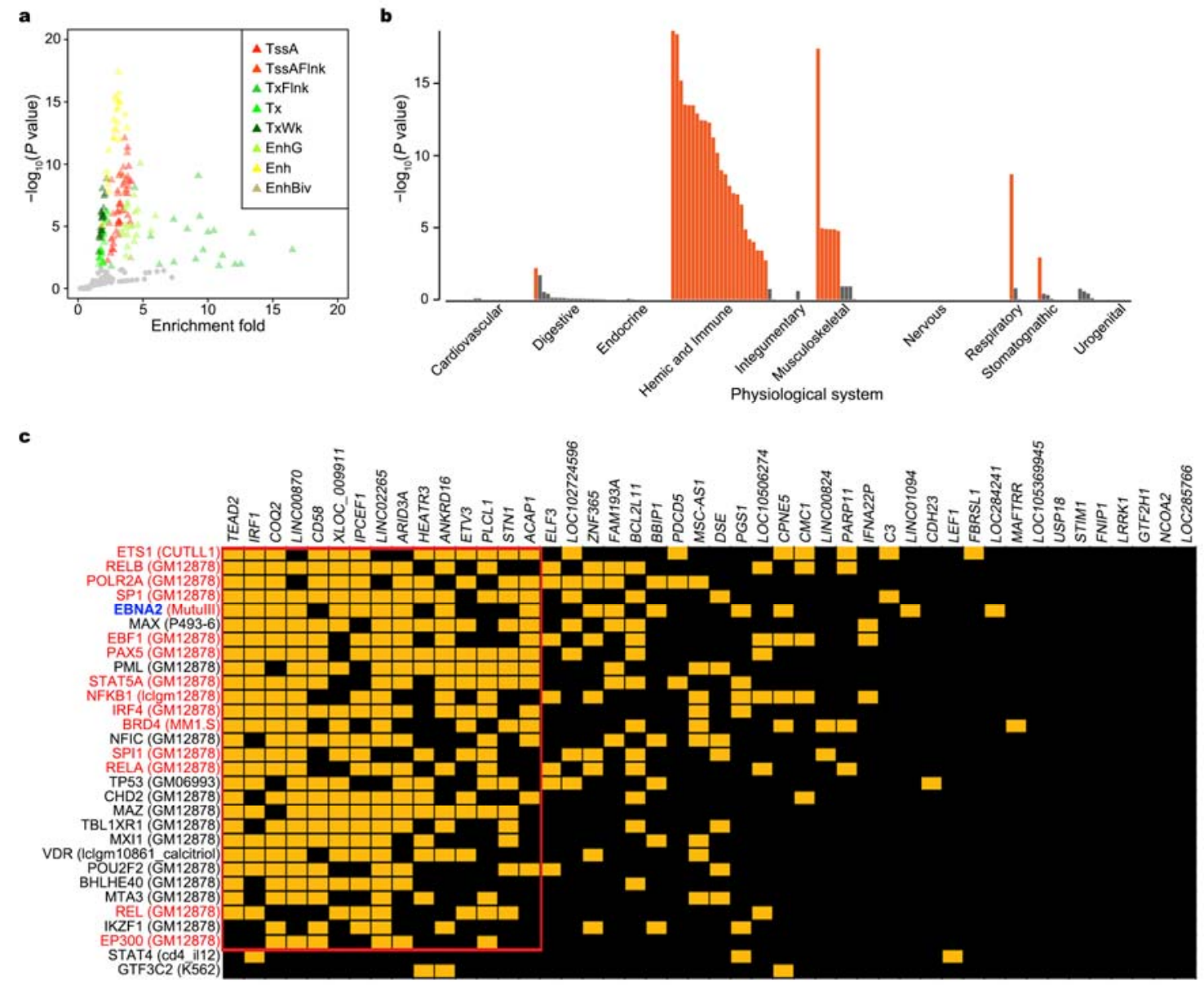

Fig. 5 | Biological interpretation of SLE associations. a, Enrichment of 112 SLEassociated non-HLA variants in ChromHMM core chromatin states across 23 immune cells. $\mathrm{X}$-axis shows fold enrichment while y-axis denotes - $\log _{10}(P$ value of enrichment). Circles and triangles indicate insignificant and significant enrichments, respectively, at FDR $<5 \%$. Chromatin states that are significantly enriched for SLE associations are labeled with their ChromHMM annotations: active transcription start site (TssA), flanking active transcription start site (TssAFlnk), transcription at gene 5' and 3' (TxFlnk), strong transcription (Tx), weak transcription (TxWk), genic enhancers (EnhG), enhancers (Enh), and bivalent enhancer (EnhBiv). b, Physiological systems implicated by the expression of genes within SLE-associated 
loci $\left(P<5 \times 10^{-8}\right)$. Physiological systems in which the expression of genes from SLEassociated loci are significantly enriched $(\mathrm{FDR}<5 \%)$ are colored orange. c, Intersection of 46 novel SLE loci with TF-DNA binding interactions. The $\mathrm{x}$-axis shows SLE loci (named after the nearest genes to the lead variants). The y-axis shows the top 36 TFs, based on probabilities obtained from RELI, sorted in descending order by the number of intersecting loci. Each intersection (yellow box) indicates that the locus (column) contains at least one SLE-associated variant located within a ChIP-seq peak for the given TF (row). The cell type resource for each of the most significant ChIP-seq datasets is indicated in parentheses. TFs that participate in EBNA2 super-enhancers are colored red. EBNA2 protein is highlighted in blue. The red rectangle identifies the optimal cluster of the locus and $\mathrm{TF}$ intersections. 


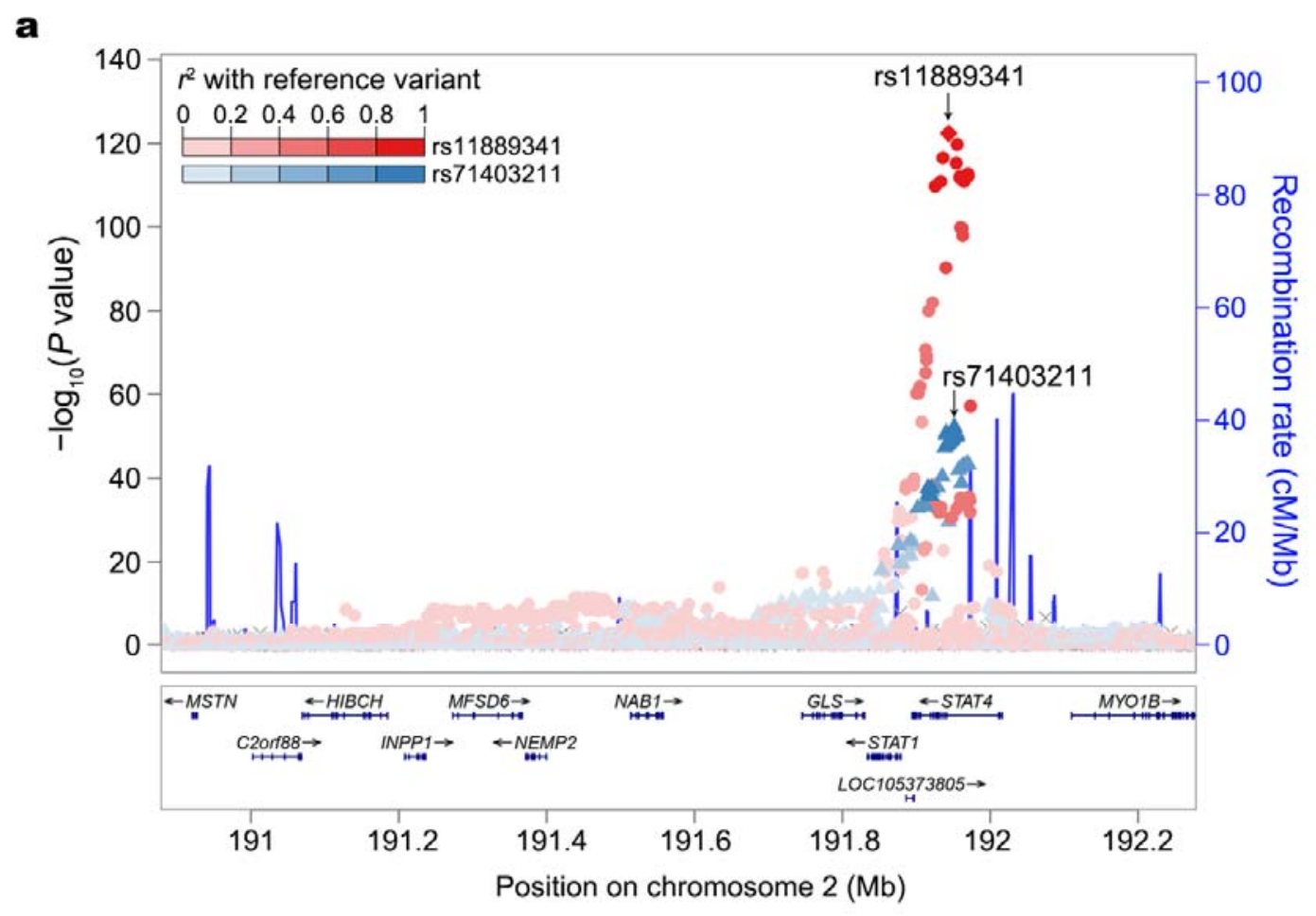

b

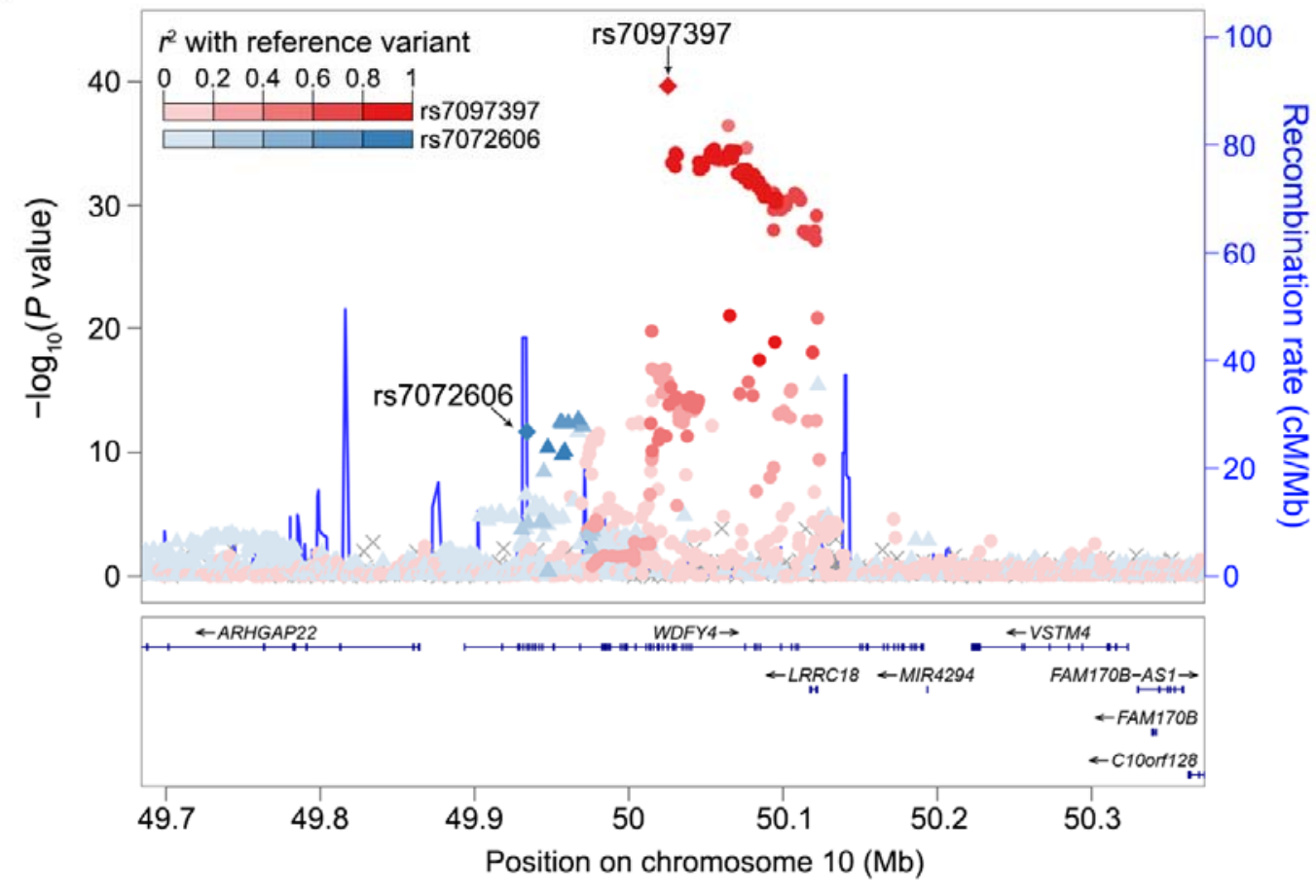

Extended Data Fig. 1| Two independent association signals identified. a, at two

intronic variants within known STAT4 locus. b, at known (rs7097397, p.Arg1816Gln) 
medRxiv preprint doi: https://doi.org/10.1101/2020.08.22.20178939; this version posted August 25, 2020. The copyright holder for this preprint (which was not certified by peer review) is the author/funder, who has granted medRxiv a license to display the preprint in perpetuity.

All rights reserved. No reuse allowed without permission.

and new (rs7072606, p.Ser214Pro) missense variants within WDFY4 locus. The lead and secondary index variants are labeled in diamond. The lead variant and its LD proxies are in red while the secondary signal index variant and its LD proxies are in blue. The LD is estimated from 7,021 Chinese samples. 

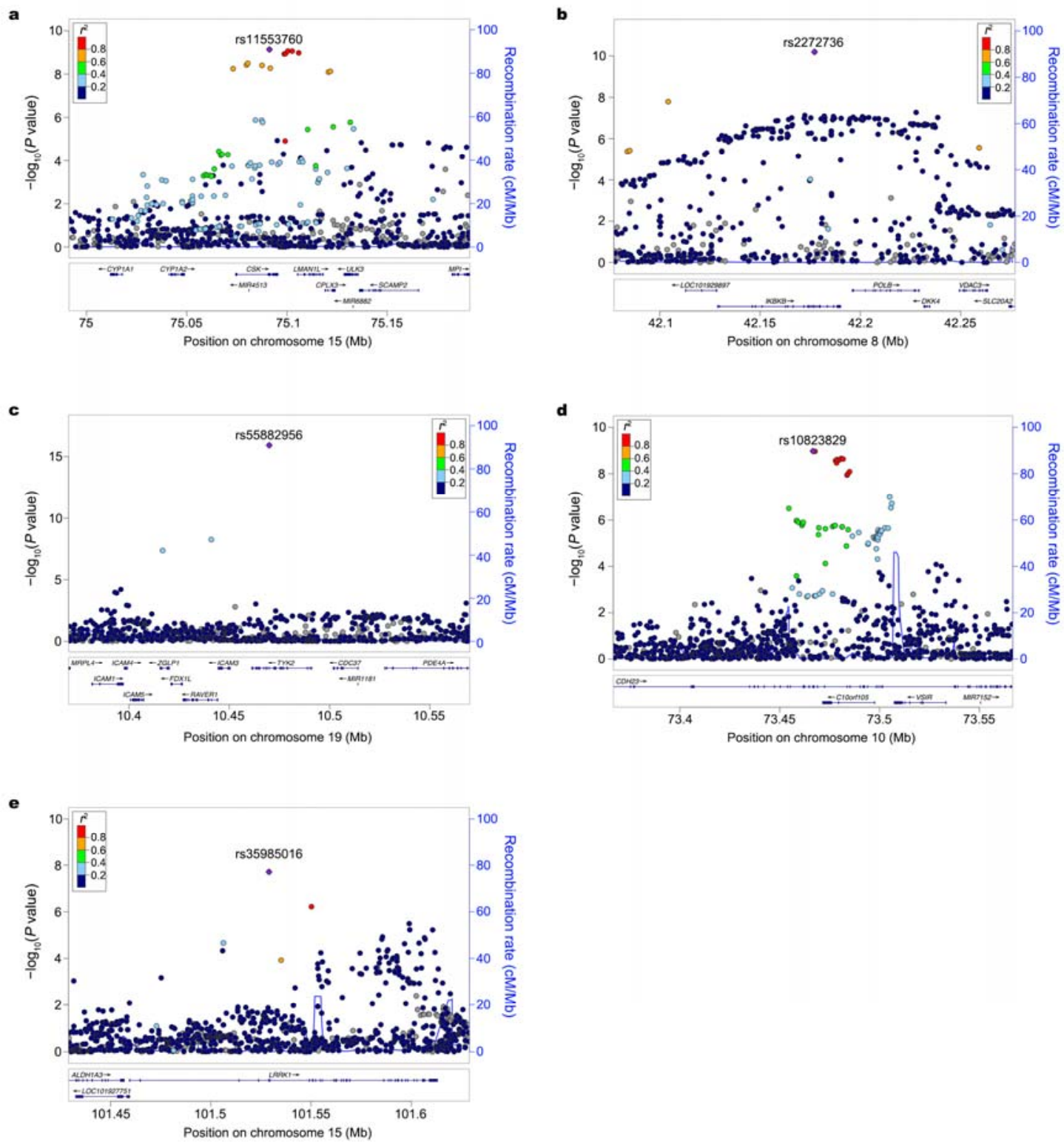

Extended Data Fig. 2 | New lead exonic variants identified at three known (CSK, IKBKB, and TYK2) and two novel (CHD23 and LRRK1) loci. a, rs11553760 (synonymous variant) at CSK. b, rs2272736 (p.Arg303Gln, missense variant) at IKBKB. c, rs55882956 (p.Arg703Trp, missense variant) at TYK2. d, rs10823829 (synonymous variant) at CHD23. e, rs35985016 (p.Lys203Glu, missense variant) at 
medRxiv preprint doi: https://doi.org/10.1101/2020.08.22.20178939; this version posted August 25, 2020. The copyright holder for this preprint (which was not certified by peer review) is the author/funder, who has granted medRxiv a license to display the preprint in perpetuity.

All rights reserved. No reuse allowed without permission.

LRRK1. The lead SNP is labeled as purple diamond. The LD is estimated from 7,021

Chinese samples. 


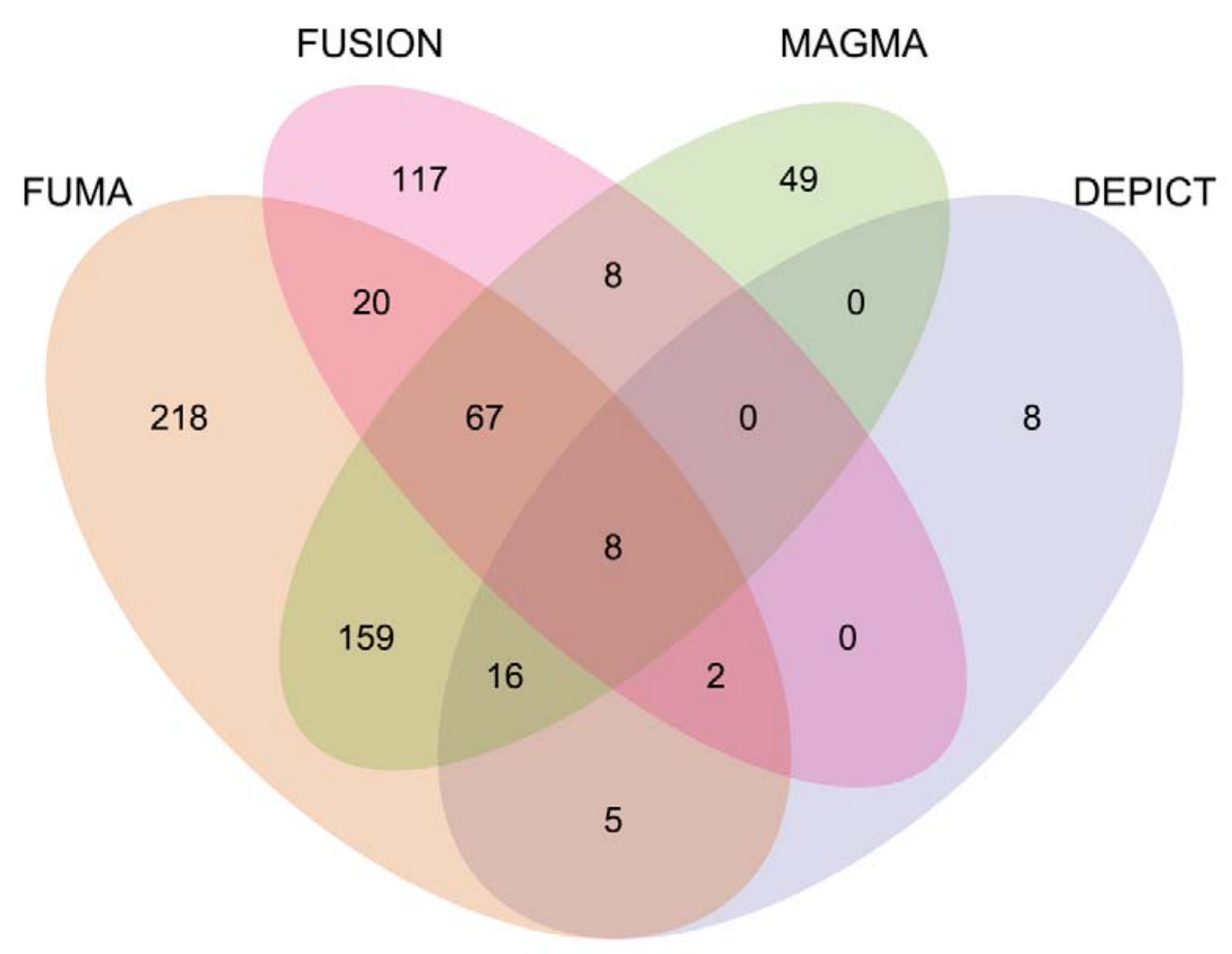

\section{Extended Data Fig. 3 | Venn diagram of significant gene findings from four}

gene-level approaches. The significant gene findings from FUMA, FUSION, MAGMA, and DEPICT are in light orange, light read, light green, and light purple, respectively. 


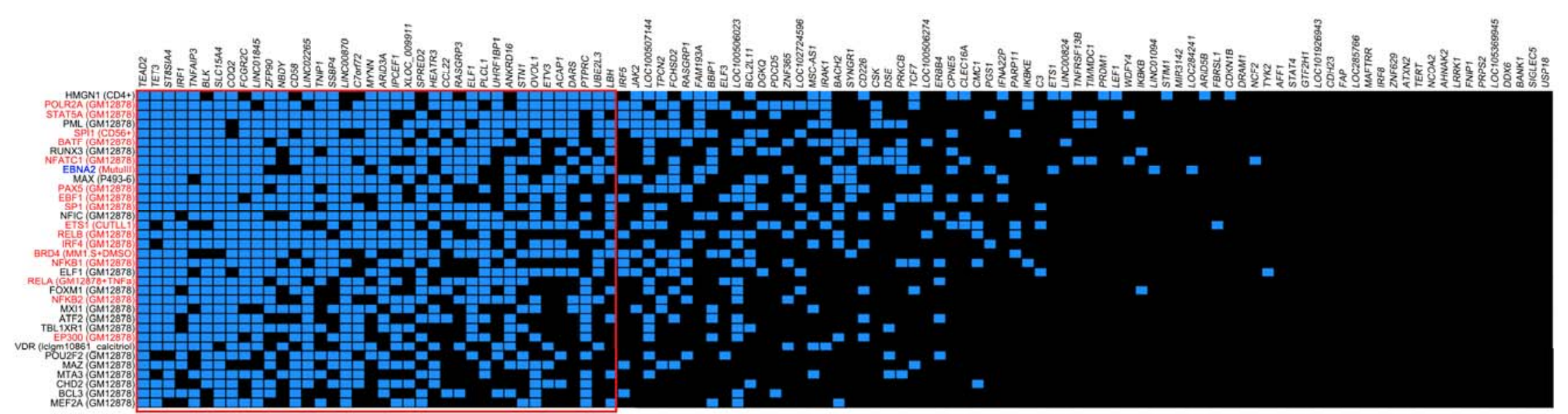

Extended Data Fig. 4 | Intersection of 112 non-HLA SLE risk loci with TF-DNA binding interactions with the genome.

The $\mathrm{x}$-axis displays SLE loci (named after the nearest genes to the lead variants). The $\mathrm{y}$-axis displays the top 36 TFs, based on probabilities obtained from RELI, sorted in descending order by the number of intersecting loci. Each intersection (yellow box) means that the locus (column) contains at least one SLE-associated variant located within a ChIP-seq peak for the given TF (row). The cell type resource for each of the most significant ChIP-seq dataset is indicated in parentheses. TFs that participate in EBNA2 super-enhancers are colored red. The red rectangle identifies the optimal cluster of the locus and TF intersections. 


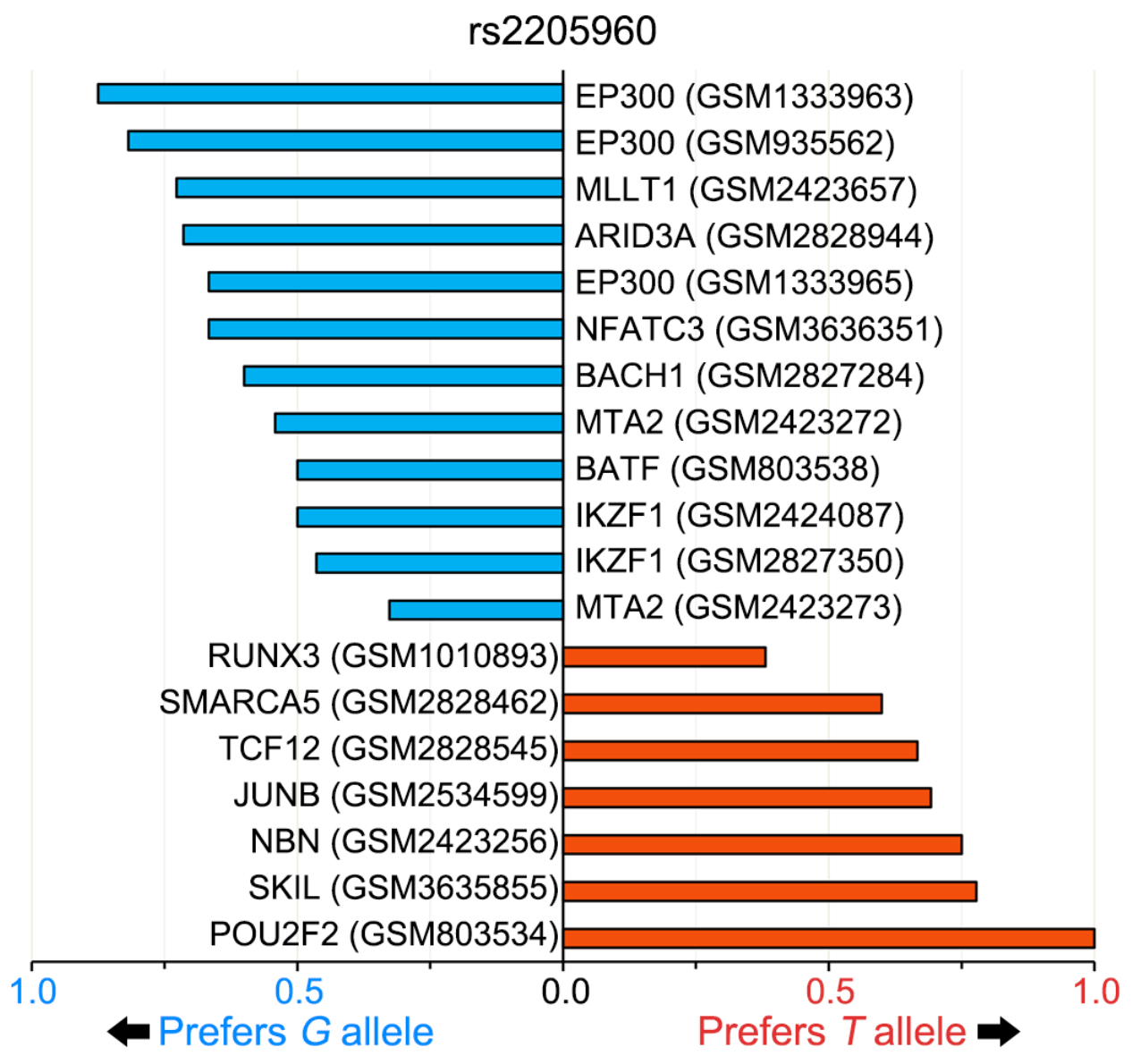

\section{Extended Data Fig. 5 | Allele-dependent regulatory proteins binding to the}

rs2205960 variant. Allele-dependent binding of regulatory proteins at the TNFSF4

locus based on ChIP-seq read allelic imbalance analysis (see Online Methods). The $\mathrm{x}$ axis indicates the preferred allele, along with a value indicating the strength of the allelic behavior, calculated as 1 minus the ratio of the weak to strong reads (for example, 0.5 indicates the strong allele has approximately twice the reads of the weak allele). 

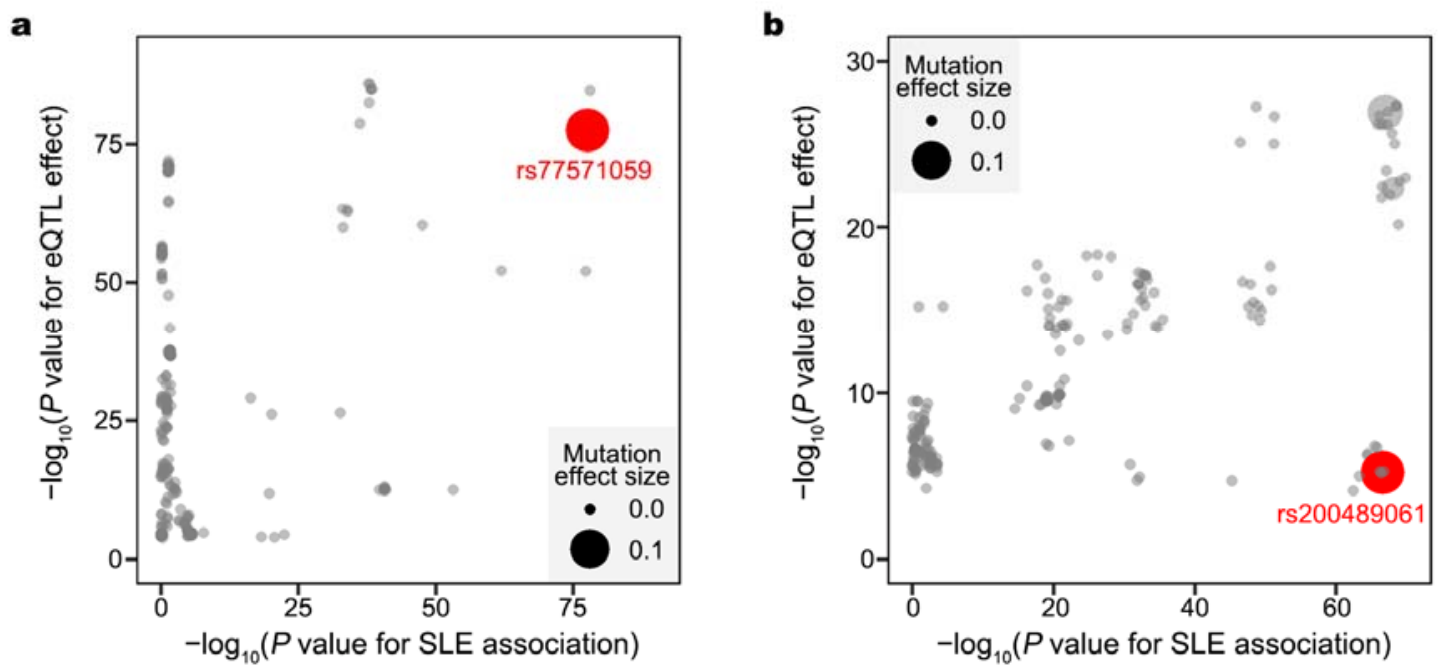

Extended Data Fig. 6 | Robust mutation effect predicted in MENTR. a, rs77571059 on regulating IRF5 expression in neutrophil. b, rs200489061 on regulating $B L K$ expression in natural killer cell. The $\mathrm{x}$-axis is the minus $\log _{10}$ transformed single-variant association P values for SLE risk while the y-axis denotes the minus $\log _{10}$ transformed eQTL P values in GTEx v8 whole blood. The size of each dot is scaled by the predicted mutation effect magnitude. The variants with robust mutation effect are in red. 
Tables

Table 1: Association results for the 46 novel susceptibility loci for SLE.

\begin{tabular}{|c|c|c|c|c|c|c|c|c|c|c|c|c|}
\hline CHR & Position & Variant & EA & NEA & EAF & OR & SE & P value & HetISq & HetPVal & $\mathbf{N}$ & Nearest gene \\
\hline 1 & $117,043,302$ & rs9651076 & $A$ & G & 0.431 & 1.117 & 0.015 & $3.26 \mathrm{E}-13$ & 10.7 & 0.347 & 208,370 & $C D 58$ \\
\hline 1 & $157,108,159$ & rs116785379 & C & G & 0.107 & 1.211 & 0.024 & $6.68 \mathrm{E}-16$ & 43.7 & 0.114 & 208,370 & ETV3 \\
\hline 1 & $201,979,455$ & rs3806357 & $A$ & G & 0.251 & 1.106 & 0.017 & $4.25 \mathrm{E}-09$ & 0.0 & 0.672 & 208,370 & ELF3 \\
\hline 2 & $7,573,079$ & rs75362385 & $\mathrm{T}$ & G & 0.321 & 0.887 & 0.017 & $8.40 \mathrm{E}-13$ & 68.3 & 0.007 & 208,370 & LOC100506274 \\
\hline 2 & $111,877,174$ & rs73954925 & C & G & 0.878 & 1.169 & 0.024 & $5.11 \mathrm{E}-11$ & 56.4 & 0.043 & 208,370 & $B C L 2 L 11$ \\
\hline 2 & $198,929,806$ & rs7572733 & $\mathrm{T}$ & C & 0.260 & 1.143 & 0.017 & $1.25 \mathrm{E}-14$ & 0.0 & 0.647 & 208,370 & PLCL1 \\
\hline 3 & $28,072,086$ & rs438613 & $\mathrm{T}$ & C & 0.588 & 0.920 & 0.014 & $7.52 \mathrm{E}-09$ & 69.4 & 0.006 & 208,370 & LINC01980 \\
\hline 3 & $72,225,916$ & rs7637844 & $A$ & C & 0.871 & 0.877 & 0.023 & $1.28 \mathrm{E}-08$ & 0.0 & 0.906 & 208,370 & LINC00870 \\
\hline 4 & $2,700,844$ & rs231694 & $\mathrm{T}$ & C & 0.380 & 1.111 & 0.018 & $9.71 \mathrm{E}-09$ & 23.7 & 0.269 & 57,253 & FAM193A \\
\hline 4 & $40,307,587$ & rs113284964 & G & GCTTC & 0.371 & 1.134 & 0.015 & $1.35 \mathrm{E}-16$ & 67.2 & 0.009 & 208,370 & LINC02265 \\
\hline 4 & $79,644,279$ & rs6533951 & $A$ & G & 0.350 & 1.111 & 0.016 & $1.25 \mathrm{E}-10$ & 61.4 & 0.024 & 208,370 & LINC01094 \\
\hline 4 & $84,146,996$ & rs6841907 & $\mathrm{T}$ & $\mathrm{C}$ & 0.729 & 0.906 & 0.016 & 1.10E-09 & 43.5 & 0.115 & 208,370 & COQ2 \\
\hline 4 & $109,061,618$ & rs58107865 & C & G & 0.227 & 0.802 & 0.021 & $6.57 \mathrm{E}-25$ & 1.1 & 0.409 & 208,370 & $L E F 1$ \\
\hline 5 & $131,120,338$ & rs370449198 & $A$ & $A C$ & 0.922 & 0.721 & 0.060 & $4.41 \mathrm{E}-08$ & 0.0 & 0.408 & 187,562 & $F N I P 1$ \\
\hline 5 & $131,829,578$ & rs2549002 & $A$ & C & 0.682 & 0.905 & 0.016 & $2.40 \mathrm{E}-10$ & 20.6 & 0.279 & 208,370 & $I R F 1$ \\
\hline 6 & 243,302 & rs9503037 & $A$ & G & 0.693 & 0.881 & 0.016 & $1.36 \mathrm{E}-15$ & 42.3 & 0.123 & 208,370 & LOC285766 \\
\hline 6 & $36,715,031$ & rs34868004 & $\mathrm{CA}$ & C & 0.225 & 1.104 & 0.017 & $4.46 \mathrm{E}-09$ & 40.7 & 0.134 & 208,370 & CPNE5 \\
\hline 6 & $116,690,849$ & rs9488914 & $\mathrm{T}$ & C & 0.920 & 0.862 & 0.026 & $1.14 \mathrm{E}-08$ & 65.3 & 0.013 & 208,370 & $D S E$ \\
\hline 6 & $154,570,651$ & rs9322454 & $A$ & G & 0.659 & 1.090 & 0.015 & $2.42 \mathrm{E}-08$ & 0.0 & 0.430 & 208,370 & $I P C E F 1$ \\
\hline 8 & $71,330,166$ & rs142937720 & $A$ & AAGTGGCC & 0.383 & 0.894 & 0.016 & $2.27 \mathrm{E}-12$ & 67.9 & 0.008 & 208,370 & NCOA2 \\
\hline 8 & $72,894,959$ & rs17374162 & $A$ & G & 0.411 & 0.917 & 0.015 & $3.02 \mathrm{E}-09$ & 35.7 & 0.169 & 208,370 & $M S C-A S 1$ \\
\hline 8 & $129,425,593$ & rs16902895 & $A$ & G & 0.678 & 1.122 & 0.016 & $1.48 \mathrm{E}-13$ & 0.0 & 0.801 & 208,370 & LINC00824 \\
\hline 9 & $21,267,087$ & rs7858766 & $\mathrm{T}$ & C & 0.538 & 1.139 & 0.016 & $2.25 \mathrm{E}-15$ & 0.0 & 0.825 & 208,370 & IFNA22P \\
\hline
\end{tabular}




\begin{tabular}{|c|c|c|c|c|c|c|c|c|c|c|c|c|}
\hline 10 & $5,910,746$ & rs77448389 & A & G & 0.913 & 0.855 & 0.025 & $7.30 \mathrm{E}-10$ & 0.0 & 0.584 & 208,370 & ANKRD16 \\
\hline 10 & $64,411,288$ & rs10995261 & $\mathrm{T}$ & C & 0.240 & 0.909 & 0.017 & $2.57 \mathrm{E}-08$ & 43.9 & 0.113 & 208,370 & ZNF365 \\
\hline 10 & $73,466,709$ & rs10823829 & $\mathrm{T}$ & C & 0.718 & 0.910 & 0.016 & $1.05 \mathrm{E}-09$ & 0.0 & 0.771 & 208,370 & $\mathrm{CDH} 23$ \\
\hline 10 & $105,677,911$ & rs111447985 & A & C & 0.073 & 1.172 & 0.028 & $1.72 \mathrm{E}-08$ & 0.0 & 0.526 & 208,370 & STN1 \\
\hline 10 & $112,664,114$ & rs58164562 & $\mathrm{T}$ & C & 0.748 & 0.892 & 0.016 & $3.14 \mathrm{E}-12$ & 33.3 & 0.186 & 208,370 & $B B I P 1$ \\
\hline 11 & $4,113,200$ & rs3750996 & A & G & 0.834 & 1.167 & 0.022 & $1.89 \mathrm{E}-12$ & 0.0 & 0.522 & 208,370 & STIM1 \\
\hline 11 & $18,362,382$ & rs77885959 & $\mathrm{T}$ & G & 0.978 & 1.694 & 0.062 & $3.16 \mathrm{E}-17$ & 0.0 & 0.511 & 204,433 & GTF2H1 \\
\hline 12 & $4,140,876$ & rs2540119 & $\mathrm{T}$ & C & 0.544 & 1.086 & 0.015 & $3.51 \mathrm{E}-08$ & 44.9 & 0.106 & 208,370 & PARP11 \\
\hline 12 & $103,916,080$ & rs6539078 & $\mathrm{T}$ & C & 0.591 & 0.894 & 0.015 & $9.49 \mathrm{E}-14$ & 0.0 & 0.916 & 208,370 & LOC105369945 \\
\hline 12 & $121,368,518$ & rs3999421 & A & $\mathrm{T}$ & 0.506 & 0.910 & 0.016 & $1.29 \mathrm{E}-09$ & 47.3 & 0.091 & 208,370 & XLOC_009911 \\
\hline 12 & $133,040,182$ & rs200521476 & G & GCATCAC & 0.812 & 0.875 & 0.023 & $5.66 \mathrm{E}-09$ & 26.7 & 0.235 & 208,370 & FBRSL1 \\
\hline 15 & $101,529,012$ & rs35985016 & A & G & 0.930 & 0.843 & 0.030 & $1.95 \mathrm{E}-08$ & 0.0 & 0.897 & 204,433 & LRRK1 \\
\hline 16 & $50,089,207$ & rs11288784 & G & GT & 0.365 & 0.902 & 0.016 & $2.38 \mathrm{E}-10$ & 0.0 & 0.664 & 208,370 & HEATR3 \\
\hline 16 & $79,745,672$ & rs11376510 & G & GT & 0.737 & 0.898 & 0.017 & $2.23 \mathrm{E}-10$ & 0.0 & 0.719 & 208,370 & MAFTRR \\
\hline 17 & $7,240,391$ & rs61759532 & $\mathrm{T}$ & C & 0.076 & 1.235 & 0.032 & $2.79 \mathrm{E}-11$ & 24.9 & 0.247 & 208,370 & $A C A P 1$ \\
\hline 17 & $47,468,020$ & rs2671655 & $\mathrm{T}$ & C & 0.651 & 1.087 & 0.015 & $4.60 \mathrm{E}-08$ & 0.0 & 0.756 & 208,370 & LOC10272459 \\
\hline 17 & $76,373,179$ & rs113417153 & $\mathrm{T}$ & C & 0.193 & 0.893 & 0.020 & $1.90 \mathrm{E}-08$ & 2.1 & 0.403 & 208,370 & PGS1 \\
\hline 18 & $77,386,912$ & rs118075465 & A & G & 0.147 & 1.140 & 0.020 & $1.16 \mathrm{E}-10$ & 0.0 & 0.543 & 208,370 & LOC284241 \\
\hline 19 & 948,532 & rs2238577 & $\mathrm{T}$ & $C$ & 0.455 & 0.885 & 0.016 & $1.83 \mathrm{E}-14$ & 60.8 & 0.026 & 208,370 & $A R I D 3 A$ \\
\hline 19 & $6,697,088$ & rs5826945 & A & $\mathrm{T}$ & 0.929 & 0.836 & 0.028 & $9.67 E-11$ & 50.0 & 0.075 & 208,370 & C3 \\
\hline 19 & $33,072,768$ & rs12461589 & $\mathrm{T}$ & C & 0.248 & 0.898 & 0.017 & $5.00 \mathrm{E}-10$ & 0.0 & 0.510 & 208,370 & PDCD5 \\
\hline 19 & $49,851,746$ & rs33974425 & CCAGCTGCAT & $C$ & 0.702 & 1.120 & 0.016 & $4.40 \mathrm{E}-12$ & 42.6 & 0.121 & 208,370 & $T E A D 2$ \\
\hline 22 & $18,649,356$ & rs4819670 & $T$ & C & 0.210 & 1.151 & 0.022 & $5.53 \mathrm{E}-11$ & 0.0 & 0.650 & 208,370 & USP18 \\
\hline
\end{tabular}

CHR: chromosome

EA: effective allele

NEA: non-effective allele 
EAF: effective allele frequency

OR: odds ratio

SE: standard error for the odds ratio

HetISq: genetic heterogeneity $I^{2}$ statistics at scale of $0-100 \%$

HetPval: P values for the chi-squared test of genetic heterogeneity

$\mathrm{N}$ : study sample size 